\title{
Administration of Maresin-1 ameliorates the physiopathology of experimental autoimmune encephalomyelitis
}

\author{
Alba Sánchez-Fernández ${ }^{1,2}$, Stephanie Zandee ${ }^{3}$, Mauricio Mastrogiovannii ${ }^{4}$ Marc Charabati ${ }^{3}$, Homero Rubbo $^{4}$, \\ Alexandre Prat ${ }^{3}$ and Rubèn López-Vales ${ }^{1,2^{*}}$
}

\begin{abstract}
Background: Resolution of inflammation is an active and regulated process that leads to the clearance of cell debris and immune cells from the challenged tissue, facilitating the recovery of homeostasis. This physiological response is coordinated by endogenous bioactive lipids known as specialized pro-resolving mediators (SPMs). When resolution fails, inflammation becomes uncontrolled leading chronic inflammation and tissue damage, as occurs in multiple sclerosis (MS).
\end{abstract}

Methods: SPMs and the key biosynthetic enzymes involved in SPM production were analysed by metabololipidomics and qPCR in active brain lesions, serum and peripheral blood mononuclear cells (PBMC) of MS patients as well as in the spinal cord of mice with experimental autoimmune encephalomyelitis (EAE). We also tested the therapeutic actions of the SPM coined Maresin-1 (MaR1) in EAE mice and studied its impact on inflammation by doing luminex and flow cytometry analysis.

Results: We show that levels of MaR1 and other SPMs were below the limit of detection or not increased in the spinal cord of EAE mice, whereas the production of pro-inflammatory eicosanoids was induced during disease progression. Similarly, we reveal that SPMs were undetected in serum and active brain lesion samples of MS patients, which was linked to impaired expression of the enzymes involved in the biosynthetic pathways of SPMs. We demonstrate that exogenous administration of MaR1 in EAE mice suppressed the protein levels of various pro-inflammatory cytokines and reduced immune cells counts in the spinal cord and blood. MaR1 also decreased the numbers of Th1 cells but increased the accumulation of regulatory $T$ cells and drove macrophage polarization towards an anti-inflammatory phenotype. Importantly, we provide clear evidence that administration of MaR1 in mice with clinical signs of EAE enhanced neurological outcomes and protected from demyelination.

Conclusions: This study reveals that there is an imbalance in the production of SPMs in MS patients and in EAE mice, and that increasing the bioavailability of SPMs, such as MaR1, minimizes inflammation and mediates therapeutic actions. Thus, these data suggest that immunoresolvent therapies, such as MaR1, could be a novel avenue for the treatment of MS.

\footnotetext{
*Correspondence: Ruben.Lopez@uab.cat

${ }^{1}$ Institut de Neurociencies and Departament de Biologia Cel lular,

Fisiologia i Immunologia, Facultat de Medicina, Universitat Autonoma de

Barcelona, 08193 Bellaterra, Catalonia, Spain

Full list of author information is available at the end of the article
}

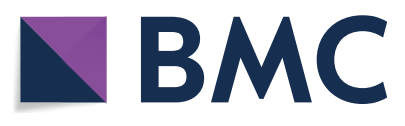

(c) The Author(s) 2022. Open Access This article is licensed under a Creative Commons Attribution 4.0 International License, which permits use, sharing, adaptation, distribution and reproduction in any medium or format, as long as you give appropriate credit to the original author(s) and the source, provide a link to the Creative Commons licence, and indicate if changes were made. The images or other third party material in this article are included in the article's Creative Commons licence, unless indicated otherwise in a credit line to the material. If material is not included in the article's Creative Commons licence and your intended use is not permitted by statutory regulation or exceeds the permitted use, you will need to obtain permission directly from the copyright holder. To view a copy of this licence, visit http://creativecommons.org/licenses/by/4.0/. The Creative Commons Public Domain Dedication waiver (http://creativeco mmons.org/publicdomain/zero/1.0/) applies to the data made available in this article, unless otherwise stated in a credit line to the data. 
Keywords: Experimental autoimmune encephalomyelitis, Inflammation, Maresin-1, Multiple Sclerosis, Resolution of inflammation, Specialized pro-resolving mediators

\section{Background}

Acute inflammation is a key physiological mechanism that promotes the repair of injured tissues and eliminates infectious organisms and toxic agents. This response is tightly controlled and typically ends with the elimination of the immune cells and cellular debris from the tissue, paving the way for the recovery of homeostasis [1-3]. Contrariwise, uncontrolled inflammation, as it occurs when the resolution phase fails, becomes harmful to the tissue, and it is a hallmark of a wide variety of pathological conditions, including in MS [2, 4-8].

For many years, resolution of inflammation was believed to be a passive process triggered by the dilution of pro-inflammatory mediators at the challenged tissue, thus halting the recruitment of leukocytes from circulation. However, it has now become clear that this process is an active and coordinated event orchestrated by a class of lipids known as SPMs [2,7]. This bioactive lipid family includes lipoxins (LX), resolvin D series (RvD), resolvin $\mathrm{E}$ series, (RvE), protectins (PD/NPD) and maresins (MaR) $[2,7,9]$. In immune cells, SPMs are naturally synthesized from omega- 3 and omega- 6 polyunsaturated fatty acids (PUFA) through a family of lipoxygenases (i.e., 12- and 15-LOX) [3, 10]. Failure to produce adequate amounts of SPMs has been associated with persistent inflammation in many inflammatory disorders, such as asthma, atherosclerosis, ulcerative colitis, spinal cord injury or Alzheimer's disease, among others $[2,4,5,11,12]$. Despite emerging data revealing that SPMs might control inflammation in central nervous system (CNS) disorders, there are very few studies addressing their importance in neuroinflammatory conditions, such as MS.

MS is a chronic neuroinflammatory and demyelinating disease of the CNS affecting around 2.5 million individuals worldwide [13-15]. Although the exact cause of MS is not yet known, a hallmark of its physiopathology is the presence of persistent numbers of immune cells in the white and grey matter of the CNS [13-16]. Indeed, neuropathological studies show that inflammation is present in all MS stages and that infiltrating leukocytes drive the formation of demyelinated and neurodegenerative lesions $[15,17,18]$.

To our knowledge, there are currently few studies addressing the contribution of SPMs in MS patients on animal models [6, 19-21]. In an early report, a connection between disease severity and SPMs production was suggested in a small cohort of MS patients since the authors found increased levels of RvD1 and NPD1 in the cerebrospinal fluid (CSF) of highly active MS patients [6]. A more recent work uncovered that progressive and relapsing-remitting MS patients had increased plasma levels of pro-inflammatory eicosanoids, whereas SPMs were reduced or undetectable [20]. Moreover, this study also found lower transcripts of the SPM biosynthetic enzymes and receptors in leukocytes from MS patients, suggesting an imbalance in peripheral induction of the resolution pathways in MS patients [20]. Interestingly, prophylactic administration of two SPM known as RvD1 and LXA4 has resulted in beneficial actions experimental autoimmune encephalomyelitis (EAE) [19, 21]. However, whether impairment in SPM production occurs in the CNS of MS patients and EAE mice, and whether administration of SPMs that are undetected in blood and CNS samples of MS patients halters inflammation and exerts therapeutic effects in animal models of MS has not been elucidated.

Herein, we reveal that there is impaired production of SPMs in active brain lesions and serum samples of MS patients, as well as in the CNS of mice undergoing EAE. We further connect these results with a defective expression of enzymes involved in the synthesis of SPMs in peripheral blood mononuclear cells (PBMCs) and in brain lesions of MS individuals, as well as, in the spinal cord of EAE mice. We also reveal that the exogenous administration of the DHA-derived SPM coined MaR1 in EAE mice once they showed the clinical signs of the disease modulates different mechanisms of inflammation and confers protection against neurological deficits and demyelination.

\section{Methods \\ MS brain tissue collection}

Autopsies on patients from Centre de Recherche du Centre Hospitalier de l'Université de Montreal diagnosed with clinical and neuropathological MS according to the revised 2010 McDonald's criteria [22] had informed consent as approved by the Centre Hospitalier de l'Université de Montréal ethics committee (BH07.001). Autopsy samples were cryopreserved, and lesions classified using Luxol Fast Blue (LFB)/Haematoxylin \& Eosin staining and Oil Red $\mathrm{O}$ staining as previously published [23, 24]. For qPCR analysis, normal-appearing white matter (NAWM) and active lesion (AL) representing 7 secondary-progressive MS (SPMS) and 2 relapsing-remitting MS (RRMS) were used. The median age at death was 50 years old (range from 26 to 65 years old) (Additional 
file 1: Table S1). Regions of interest (lesion or NAWM) for RNA isolation were dissected manually from 5 to $650 \mu \mathrm{m}$ cryosections per block. For lipidomic analysis, normalappearing white matter (NAWM) and active lesions (AL) representing 6 secondary-progressive MS (SPMS) and 2 relapsing-remitting MS (RRMS) were used. The median age at death was 50 years old (range from 26 to 65 years old) (Additional file 1: Table S2).

\section{MS and healthy donor PBMC and serum collection}

For isolation of the peripheral blood mononuclear cells (PBMCs), blood samples were collected from 8 patients with relapsing-remitting MS (RRMS) and 3 with secondary-progressive MS (SPMS), recruited at the MS Clinic at the Centre de Recherche du Centre Hospitalier de l'Université de Montréal. The mean age was 43 years old (ranged from 27 to 65) (Additional file 1: Table S1). 8 healthy volunteers were included as controls, whose mean age was 35 years old (range from 24 to 42 years old) (Additional file 1: Table S1). PBMCs were isolated from blood samples collected in EDTA-coated Vacutainer tubes (BD Biosciences, Oakville, ON, Canada), using a Ficoll density gradient as previously described [25].

Moreover, serum samples were also obtained from 10 patients with RRMS and 10 healthy volunteers. The mean age was 47 years old (ranging from 36 to 55 ) and 43 years old (ranging from 35 to 55) in the MS and healthy donor group, respectively (Additional file 1: Table S2).

A written informed consent was obtained from patients and healthy donors in accordance with the local ethics committee (CRCHUM research ethic committee approval number BH07.001).

\section{Experimental autoimmune encephalomyelitis}

All experimental procedures were approved by the Universitat Autònoma de Barcelona Animal Experimentation Ethical Committee (CEEAH 2878) and followed the European Communities Council Directive 2010/63/EU, and the methods were carried out in accordance with the approved guidelines.

Female adult C57Bl/6 (8-10 weeks old; Charles River Laboratories) were sedated with intramuscular injection of a mixture of ketamine $(22 \mathrm{mg} / \mathrm{kg}$ ) (Imalgen 1000, Merial) and xylazine $(2.5 \mathrm{mg} / \mathrm{kg}$ ) (Rompun, Bayer). Experimental autoimmune encephalomyelitis (EAE) was actively induced by subcutaneously immunization with $300 \mu \mathrm{g}$ of myelin oligodendrocyte glycoprotein peptide 35-55 (MOG ${ }_{35-55}$ MEVGWYRSPFSRVVHLYRNGK, Thermo Fisher Scientific, MA, USA) in $200 \mu \mathrm{L}$ Complete Freund's Adjuvant (CFA) (Difco, MI, USA) supplemented with $4 \mathrm{mg} / \mathrm{mL}$ of heat inactivated Mycobacterium tuberculosis (Difco, MI, USA). Intraperitoneal (i.p.) injections of $400 \mathrm{ng}$ of pertussis toxin (Sigma-Aldrich, ON, USA) in $100 \mu \mathrm{L}$ sterile saline were administered at the time of induction and again $48 \mathrm{~h}$ later. All the mice were housed with food and water ad libitum at a room temperature of $22 \pm 2^{\circ} \mathrm{C}$ under $12: 12 \mathrm{~h}$ light-dark cycle. EAE onset was considered on the day animals showed the first signs of disease (EAE score 0.5 or 1 ; around day 9-10 post-immunization). Disease peak was considered on the day EAE score did not increase from the previous day (day 16-18 post-immunization). Chronic phase of the disease was considered at day 21 post-immunization if EAE score decreased or was maintained with respect to disease peak.

\section{EAE functional evaluation}

Mice were daily scored from day 0 to day 21 after induction of EAE. The researcher was blind to experimental groups during the functional evaluation. A 6-point scale was used to evaluate the clinical signs of EAE: $0=$ normal walking, $0.5=$ partially paralysed tail, $1=$ fully paralysed tail, $2=$ mild hind limb weakness, quick righting reflex, $3=$ severe hind limb weakness, slow righting reflex, unable to bear weight, $3.5=$ severe hind limb weakness, partial paralysis of hind limb, $4=$ complete paralysis of at least one hind limb, $4.5=$ complete paralysis of one or both hind limbs and trunk weakness, $5=$ complete paralysis of one or both hind limbs, forelimb weakness or paralysis, and $6=$ moribund.

\section{Drug administration}

EAE-induced mice were randomly assigned to the treatment and control experimental groups. Daily injections (i.p.) of $1 \mu \mathrm{g}$ of MaR1 (7R,14S-dihydroxy-4Z, 8E, 10E,12Z, 16Z, 19Z-DHA; Cayman Chemical)) in $200 \mu \mathrm{L}$ of sterile saline $(0.9 \% \mathrm{NaCl})$ or vehicle were initiated in each individual mouse the first day of the clinical signs until the end of the study 21 days after induction. The dose of MaR1 was chosen based on the effectivity of this SPM in spinal cord injury [4].

\section{Real-time quantitative PCR assay (qPCR)}

Unimmunized and immunized female C57Bl6/J mice at different stages of the disease (onset: 11/12 days after EAE induction, peak: 17 days after EAE induction, and chronic phase: 21 days after EAE induction) were transcardially perfused with $60 \mathrm{~mL}$ of sterile saline $(0.9 \%$ $\mathrm{NaCl}$ ) and lumbar spinal cords were harvested. Total RNA was isolated using RNeasy Lipid Tissue Mini Kit (Qiagen) according to the manufacturer's instruction.

RNA was isolated from post-mortem MS brain samples and PBMCs from MS patients and healthy donors using RNeasy Lipid Tissue Micro Kit (Qiagen) and QIAamp RNA Blood Tissue Mini Kit (Qiagen), respectively, according to the manufacturer's guidelines. 
$1 \mu \mathrm{g}$ of RNA isolated from mouse spinal cords and human PBMCs, and $0.1 \mu \mathrm{g}$ of RNA from human brain samples, was then retrotranscribed using cDNA Reverse Transcription Kit (Applied Biosystems). qPCR was performed using TaqMan reagents from Applied Biosystems and the following TaqMan-designed primers (Thermo Fisher Scientific): mouse Alox-12/15 (Mm00507789_m1), human ALOX12 (Hs00167524_m1) and human ALOX-15 (Hs00993765_g1). Mouse Gapdh (Mm99999915_g1) and human GAPDH (Hs02786624_g1) were used as housekeeping gene. qPCR was performed using the Bio-Rad CFX384 apparatus and software. The amount of cDNA was calculated based on the threshold cycle $(C T)$ value and was standardized by the amount of Gapdh using the $2^{-\Delta \Delta C T}$ method [26].

\section{Lipidomic analysis}

Lipidomic analysis was done on brain AL and NAWM tissue obtained from MS patients, serum samples collected from MS and healthy volunteers and spinal cord samples harvested from both naïve and EAE mice at different stages of the disease (onset, peak and chronic phase).

All samples were thawed and weighted and $180 \mu \mathrm{L}$ $\mathrm{MeOH}$ containing internal deuterated standard mix was added. Homogenization was performed with zirconium oxide beads in a bullet blender (Next Advance) according to the manufacturer's instructions and further centrifugation at $14.000 \mathrm{~g}$ was done. Supernatants were diluted with $0.1 \%$ formic acid (1:9) and oxylipin analysis was performed. Human serum samples $(500 \mu \mathrm{L})$ were thawed and diluted in equal volume of $20 \% \mathrm{MeOH}$ containing internal deuterated standard mix. After centrifugation at $14.000 \mathrm{~g}$, supernatants were submitted to oxylipin analysis as described below.

Oxylipin analysis was performed as previously described [27]. Briefly, samples are transferred to preconditioned Strata $^{\mathrm{TM}}-\mathrm{X}$ SPE cartridges (Phenomenex, Torrance, $\mathrm{CA}$ ) and washed with $10 \% \mathrm{MeOH}$ solution in acidified water $(\mathrm{HCl}, \mathrm{pH} 3)$. Then oxylipins were eluted with $\mathrm{MeOH}$ under vacuum, dried and reconstituted with $50 \% \mathrm{MeOH}$. Extracts were separated on a Luna C18(2) column (Phenomenex, Torrance, CA) and analysed by negative mode electrospray ionization using scheduled multiple reaction monitoring (sMRM) on a spectrometer QTRAP4500 (ABSciex, Framingham, MA). Identification and quantitation assessed by internal standard methods were performed with Analyst 1.6.2 software (ABSciex).

\section{Histological analysis}

EAE mice were euthanized on day 21 post-immunization with an overdose of pentobarbital sodium (Dolethal) and transcardially perfused with $4 \%$ paraformaldehyde (PFA) in $0.1 \mathrm{M}$ phosphate buffer (PB). Lumbar segments of spinal cords were harvested, post-fixed in 4\% PFA for $2 \mathrm{~h}$ and cryoprotected in $30 \%$ sucrose in $0.1 \mathrm{M}$ at $4{ }^{\circ} \mathrm{C}$ for at least $48 \mathrm{~h}$. Spinal cords were embedded in TissueTek OCT (Sakura), cut into transversal Section (15 $\mu \mathrm{m}$ thick) with a cryostat (Leica) between L3 and L5 segments and serially picked up on gelatine-coated glass slides. Samples were stored at $-20{ }^{\circ} \mathrm{C}$.

To measure the myelinated area, sections were stained with LFB (Sigma-Aldrich). Briefly, after a graded dehydration, sections were placed in $1 \mathrm{mg} / \mathrm{mL}$ of LFB solution in $96 \% \mathrm{EtOH}$ and $0.05 \%$ acetic acid overnight at $37{ }^{\circ} \mathrm{C}$ and protected from light. Then, slides were washed with $96 \% \mathrm{EtOH}$, rehydrated in distilled water and placed in a $0.5 \mathrm{mg} / \mathrm{mL} \mathrm{Li}_{2} \mathrm{CO}_{3}$ solution for 3-5 min at room temperature. Finally, sections were washed in distilled water, dehydrated again in 100\% $\mathrm{EtOH}$ and mounted in DPX (Sigma-Aldrich). To assess the demyelinated area in the spinal cord, 6 random images per mouse were captured at $10 \mathrm{X}$ magnification with an Olympus BX51 and the attached Olympus DP73 Camera. Demyelination was assessed by measuring the demyelinated areas within the spinal cord white matter. These analyses were done using the Image J analysis software.

To analyse axonal damaged, sections were incubated overnight at $4{ }^{\circ} \mathrm{C}$ with an antibody against neurofilament (1:1000; Millipore). Next, sections were washed twice in phosphate-buffered saline (PBS) and incubated for $1 \mathrm{~h}$ at room temperature with the secondary antibody donkey anti-rabbit Alexa Fluor 594 (1:500; Invitrogen) and washed again in PBS and PB. Finally, sections were stained with Dapi (1:1000; Sigma-Aldrich) to label nuclei and mounted in DPX (Sigma-Aldrich) after a graded dehydration. To analyse the samples, random images were captured at $40 \mathrm{X}$ magnification with an Olympus BX51 and the attached Olympus DP73 Camera.

\section{Cytokine expression}

EAE mice were euthanized at peak of the disease (17 days after EAE induction) with an overdose of pentobarbital sodium (Dolethal) and transcardially perfused with $60 \mathrm{~mL}$ of sterile saline $(0.9 \% \mathrm{NaCl})$. Spinal cords were harvested and rapidly frozen in liquid nitrogen. Protein isolation from the spinal cord samples and cytokine quantification were performed as described previously [28]. The protein levels of the following cytokines (IL-4, IL-10, IL-1 $\alpha$, IL-1 $\beta$, IL-3, IL-6, TNF- $\alpha$, IFN- $\gamma$, IL-17A, CSF-3, CCL-5, CCL-2, CXCL-2 and CXCL-10) were analysed using a custom-designed Cytokine Magnetic Bead Panel (Invitrogen) on a MAGPIX system (Millipore). 


\section{Flow cytometry}

Immune cell infiltration was determined from blood, lymph nodes and spinal cord of EAE mice at the peak of EAE (17 days after EAE induction). Briefly, mice were euthanized with an overdose of pentobarbital sodium (Dolethal). $15 \mu \mathrm{L}$ of blood was collected from a cardiac puncture and stored in heparinized vials at $4{ }^{\circ} \mathrm{C}$. Then, mice were transcardially perfused with $60 \mathrm{~mL}$ of sterile saline $(0.9 \% \mathrm{NaCl})$ and the spinal cords and lymph nodes (cervical and inguinal) were collected.

Blood samples were incubated with red blood cell lysis buffer (BioLegend) according to manufactures' guide to obtain a cell suspension enriched in leukocytes. Spinal cords and lymph nodes were cut into small pieces and enzymatically dissociated in $1 \mathrm{~mL}$ of Hank's Balanced Salt Solution (HBSS; Sigma-Aldrich) without $\mathrm{Ca}^{2+} / \mathrm{Mg}^{2+}$ containing $0.1 \%$ collagenase and $0.1 \%$ DNase for $30 \mathrm{~min}$ at $37{ }^{\circ} \mathrm{C}$, and then, mechanically disintegrated through a $70-\mu \mathrm{m}$ cell strainer to obtain a cell suspension [28].

Cell suspensions were split into different $1.5 \mathrm{~mL}$ microcentrifuge tubes according to the number of antibody combinations. For extracellular staining, samples were split, and unstained cells and isotype-matched control samples were generated to control for nonspecific binding of antibodies and for autofluorescence. For surface staining the following antibodies from eBioscience were used at a 1:300 concentration: CD45-PerCP, CD11b-PE or PE-Cy7, F4/80-PE or -APC, Ly6C-FITC, Ly6G-PE, CD3-FITC-APC-PerCP; CD4-APC-Cy7, CD8APC, CD49b-PE, CD24-PE, CD16/32 PE, CD206 FITC, CD11a-PE, CD49d-FITC, ICAM-1-FITC and CD62LPE-Cy7. Samples were incubated with the primary antibodies for $1 \mathrm{~h}$ at $4{ }^{\circ} \mathrm{C}$ with gentle agitation, washed with Dulbeccos' Modified Eagle Medium (DMEM)-10\% foetal bovine serum (FBS) and centrifuged twice at $300 \mathrm{~g}$ for $10 \mathrm{~min}$ at $4{ }^{\circ} \mathrm{C}$ to remove debris and then fixed with $1 \%$ PFA.

For intracellular staining in those samples where required, the following antibodies were also used at 1:300 concentration: FoxP3- PE-Cy7, tBet-PerCP, ROR $\gamma$ APC, GATA3-PE, IFNy-Alexa488, IL-17A-Alexa488, IL-4-APC, IL-10-Alexa488 or APC (all of them from eBioscience) iNOS-Alexa 647 (Abcam) and Arg1-Alexa 488 (Santa Cruz). Cells were fixed and permeabilized with FoxP3 Transcription Factor Staining Buffer Set (eBioscience). Samples were immunostained with the intracellular antibodies for overnight at $4^{\circ} \mathrm{C}$. In the case cytokines antibodies, samples were incubated $4 \mathrm{~h}$ at $37{ }^{\circ} \mathrm{C}$ in agitation with Cell Stimulation Cocktail 1X (eBioscience) and Brefeldin A $10 \mu \mathrm{L} / \mathrm{ml}$ (Biolegend) before the fixation and permeabilization for intracellular staining. Finally, stained cells were washed with PBS twice and fixed with $1 \%$ PFA. Samples were analysed on a FACS Canto Flow
Cytometer (BD Bioscience) and all data were processed using FlowJo ${ }^{\circledR}$ software V.10. Microglia cells were defined as $\mathrm{CD} 45^{\text {low }}$ and $\mathrm{CD} 11 \mathrm{~b}^{+}$, whereas macrophages were defined as $\mathrm{CD} 45^{\text {high }}, \mathrm{CD}_{11} \mathrm{~b}^{+}$and $\mathrm{F} 4 / 80^{+}$and granulocytes (mainly neutrophils) as CD45 $5^{\text {high }}, \mathrm{CD}_{11 \mathrm{~b}^{+}}$and F4 $/ 80^{-}$according to previous publications $[4,27]$

\section{Statistical analyses}

Data are shown as mean \pm standard error of the mean (SEM). The Kolmogorov-Smirnov test was used to check whether the data was normal distributed. Two-tailed Student's $t$ test was used for the comparison between two different groups (qPCR, histological analysis, normalized cytokine levels and flow cytometry analysis). EAE clinical score was analysed by using two-way repeated measures ANOVA with post hoc Bonferroni's test for multiple comparisons. Cytokine levels expressed in picograms/ grams of protein in four different groups were analysed by using a one-way ANOVA with post hoc Tukey's test for multiple comparison. Differences were considered significant at $p<0.05$.

\section{Results}

There is an imbalance between pro-inflammatory and pro-resolving lipid mediators in EAE mice

We first conducted metabolomic analysis in spinal cord samples of EAE mice to study whether the production of SPMs was induced in the CNS of one of the most widely used animal models of MS. We found that levels of DHA dropped in the spinal cord of EAE, especially at chronic stages of the disease, although this reduction did not reach statistical significance (Fig. 1A).

Further analysis of DHA metabolites revealed some DHA mediators produced by $12 / 15 \mathrm{LOX}$, such as 14HDoHA and 17HDoHA, which are pathway markers for the formation of Maresins, RvD series and Protectin D1 (PD1), tended to increase at these EAE stages, but not at the significant level (Fig. 1A). Although PD1, RvD1 and RvD5 were detected in the spinal cord at physiological conditions, their levels did not increase in any of the EAE stages analysed (Fig. 1A). Other SPMs derived from DHA, such as MaR1, MaR2 and some members from the RvD series, such as RvD2, Rv3 and Rv4, were undetected in the mouse spinal cord at physiological conditions and during EAE disease. The only DHA metabolite that was significantly augmented in EAE mice was the 16(17)-EpDPE, which is produced via cytochrome P450 (CYP450) and has unknown functions (Fig. 1A).

Levels of EPA remained altered in the spinal cord of EAE mice, although they tended to be higher at chronic stages of the disease (Fig. 1B). However, lipid mediators generated from EPA by 12/15LOX, such as 12-HEPE and 15-HEPE, were increased in the spinal cord of EAE, 
A

DHA metabolites
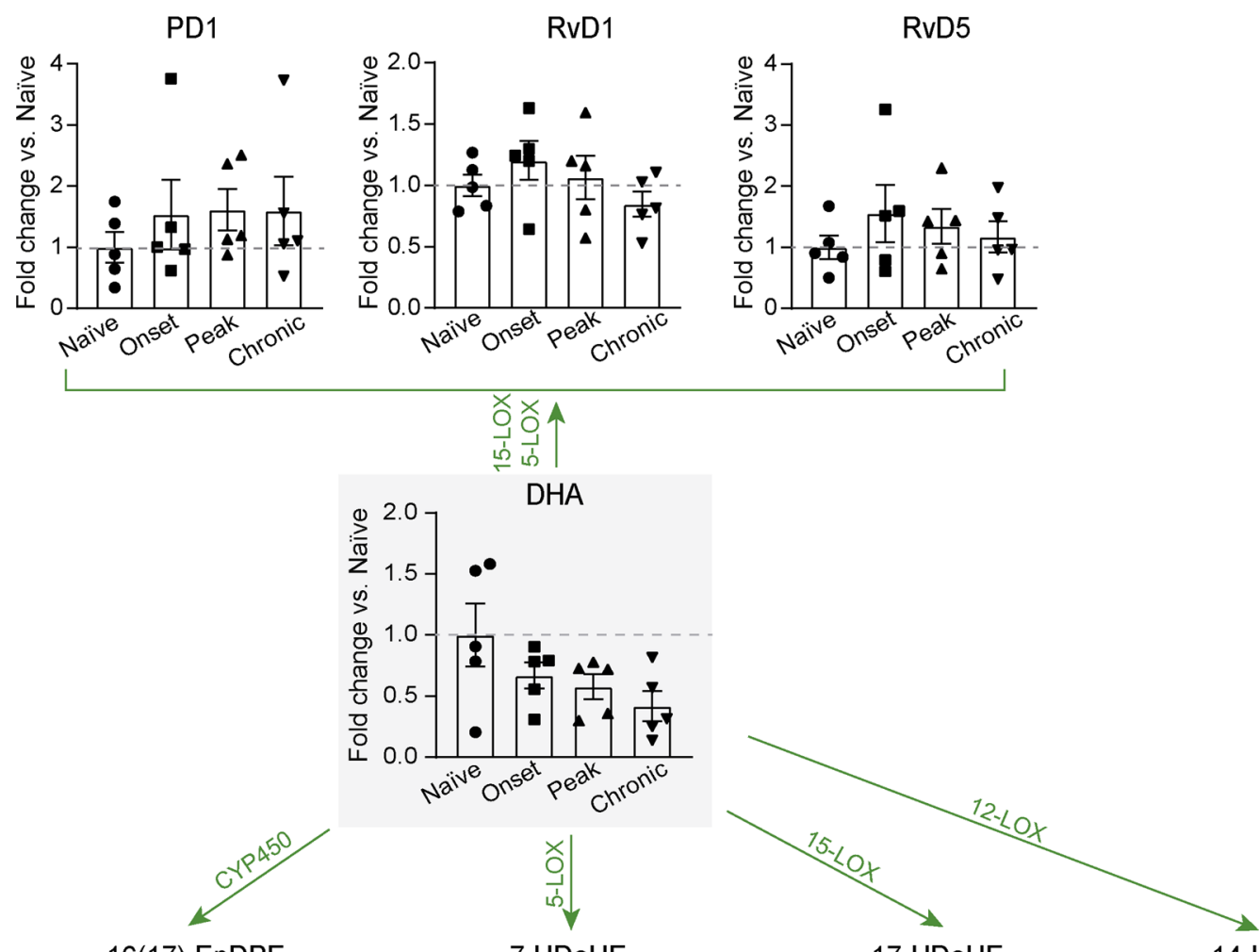

16(17)-EpDPE
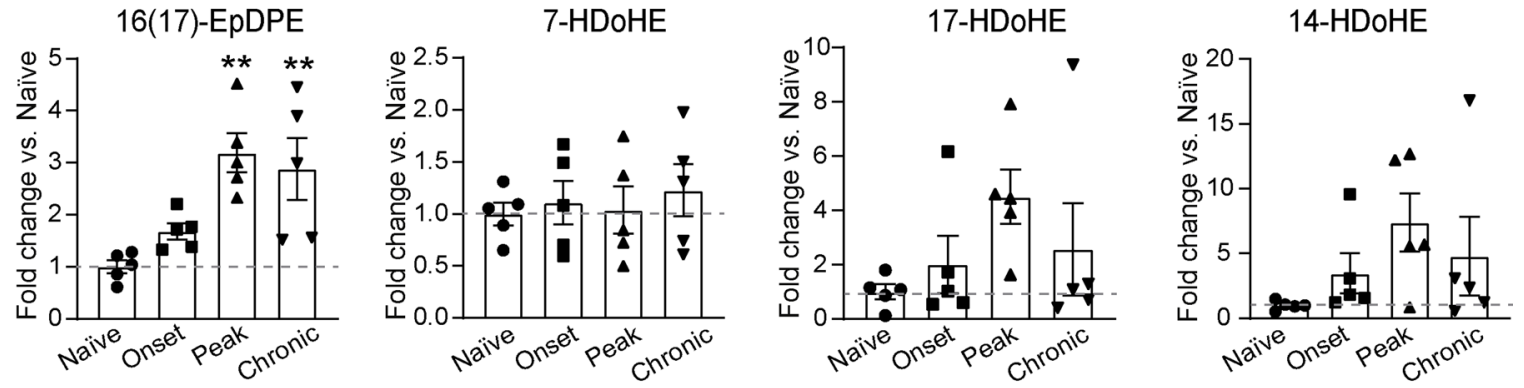

B

\section{EPA metabolites}
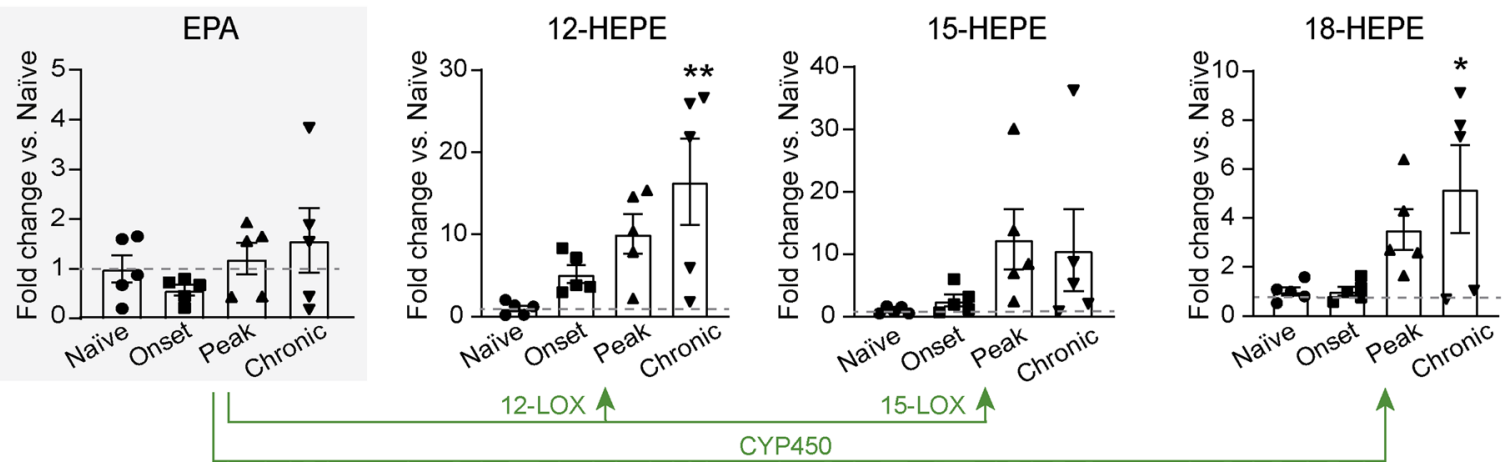

Fig. 1 Lipidomic analysis of SPM synthesis pathways markers in the CNS of naive and EAE mice. A, B Graphs showing the quantification of $\mathbf{A}$ DHA metabolome and $\mathbf{B}$ EPA metabolome in the spinal of naïve and EAE mice ( $n=5$ per group). Dotted line highlights the naïve value. ${ }^{*} p<0.05$; ${ }^{* *} p<0.01$; vs. vehicle. Two-way ANOVA with repeated measures, Bonferroni's post hoc test. Data are shown as mean \pm SEM 
especially at the chronic phase of the disease, but only 12-HEPE levels reached statistical significance (Fig. 1B). The levels of 18-HEPE, the RvE-series lipid intermediate produced via CYP450, were significantly increased in the spinal cord of chronic EAE (Fig. 1B). However, none of the members of the RvE series were detected.

As for the AA metabolome, we found that the levels of the precursor (AA) were reduced in the spinal cord of EAE mice, reaching statistical significance at chronic stages of the disease (Fig. 2A). However, we found a significant increase in the levels of 11,12-EET, which is generated by CYP450, at the peak and chronic phase of EAE disease (Fig. 3C). Lipid mediators generated from AA by 12/15LOX, such as 12-HETE and the intermediate metabolite in LXA4 and LXB4 synthesis, 15-HETE, were also increased in the spinal cord of EAE mice. However, significant differences were only observed in 15-HETE levels at chronic stages of the disease (Fig. 2), while LXA4 and LXB4 were undetected.

In contrast to SPMs, most of the pro-inflammatory lipid mediators derived from AA, such as LTB4, PGD2, PGE2, PGF2 and TXB2, were significantly increased in the spinal cord of EAE mice at the peak and/or chronic stage (Fig. 2).

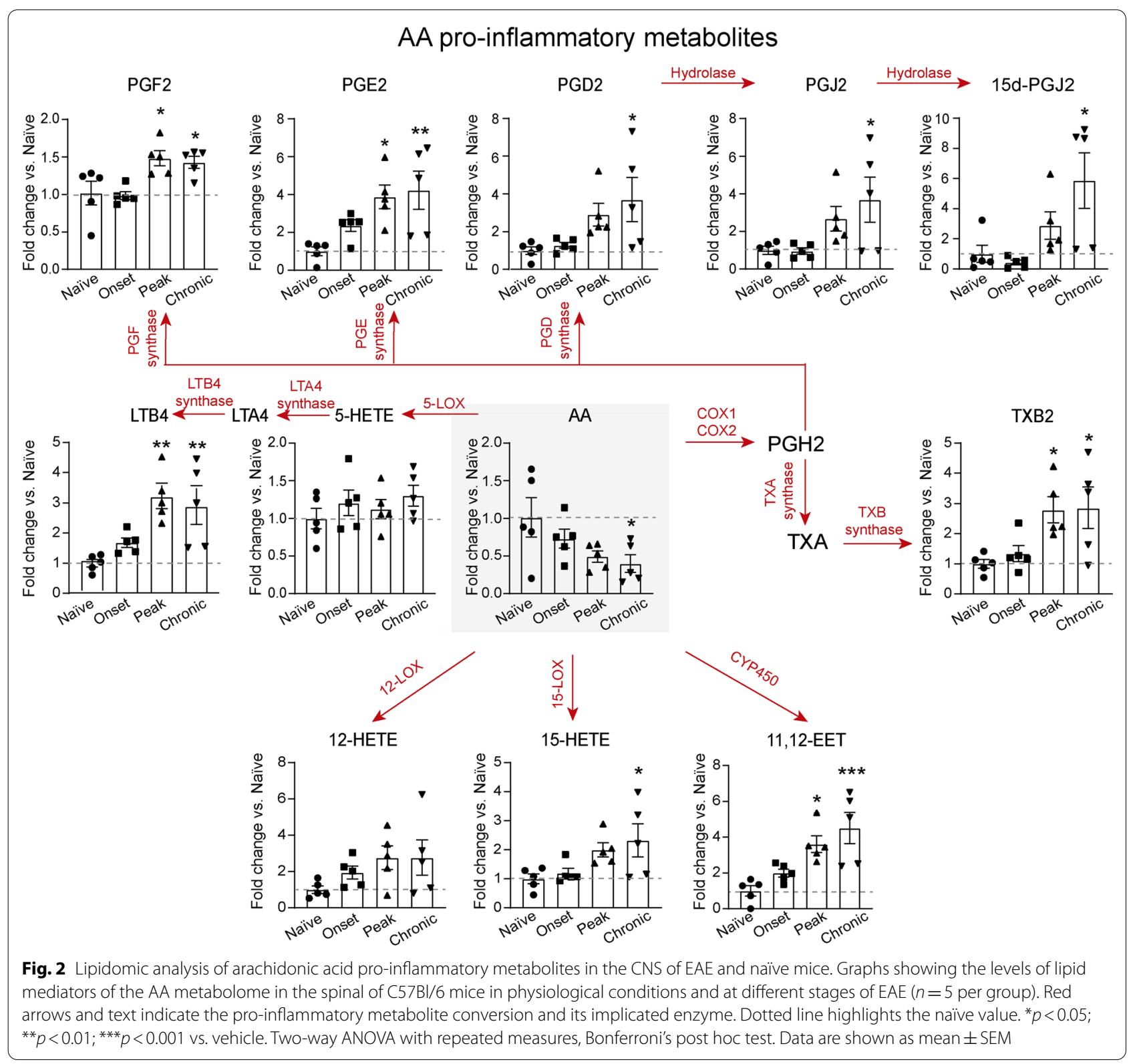




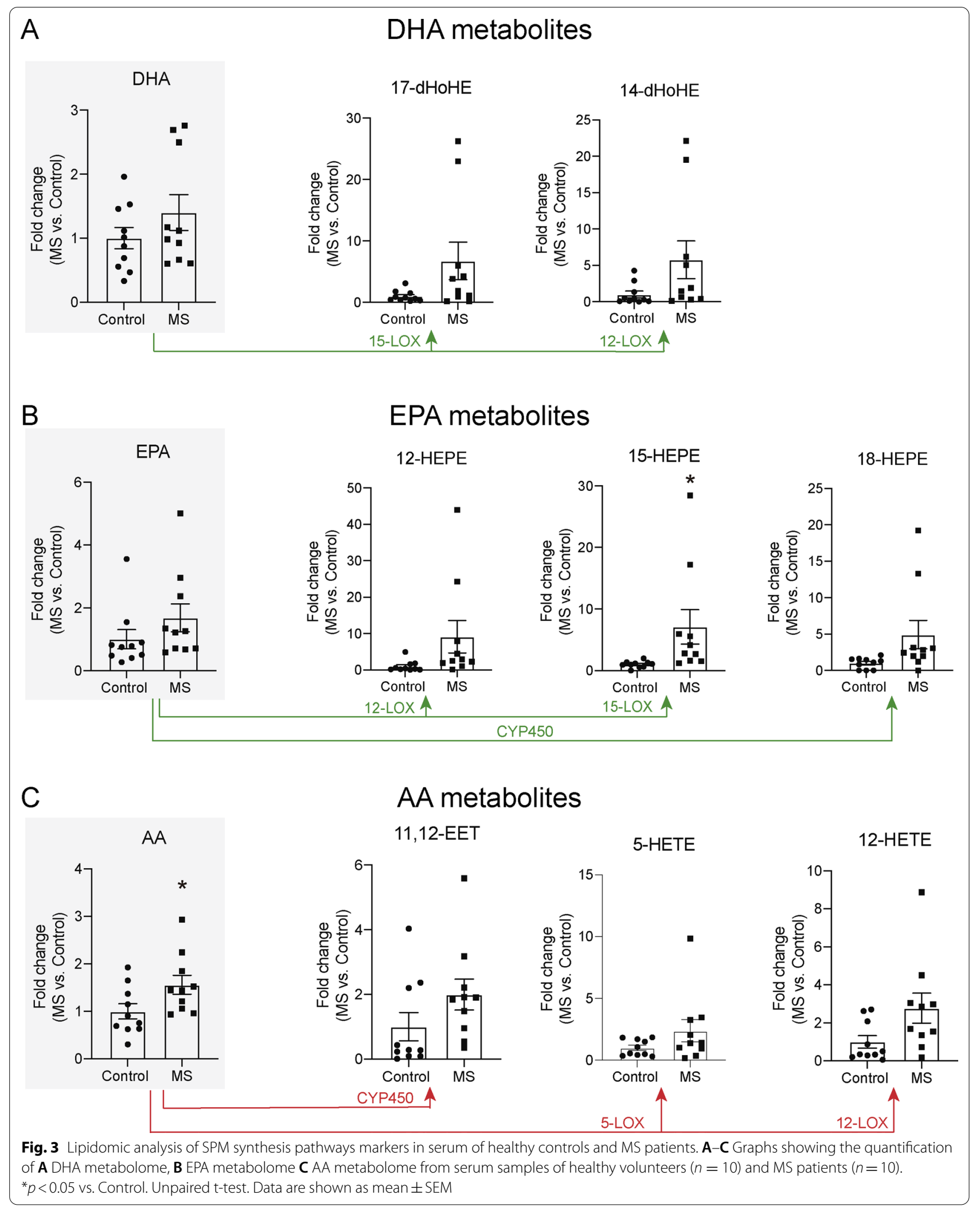




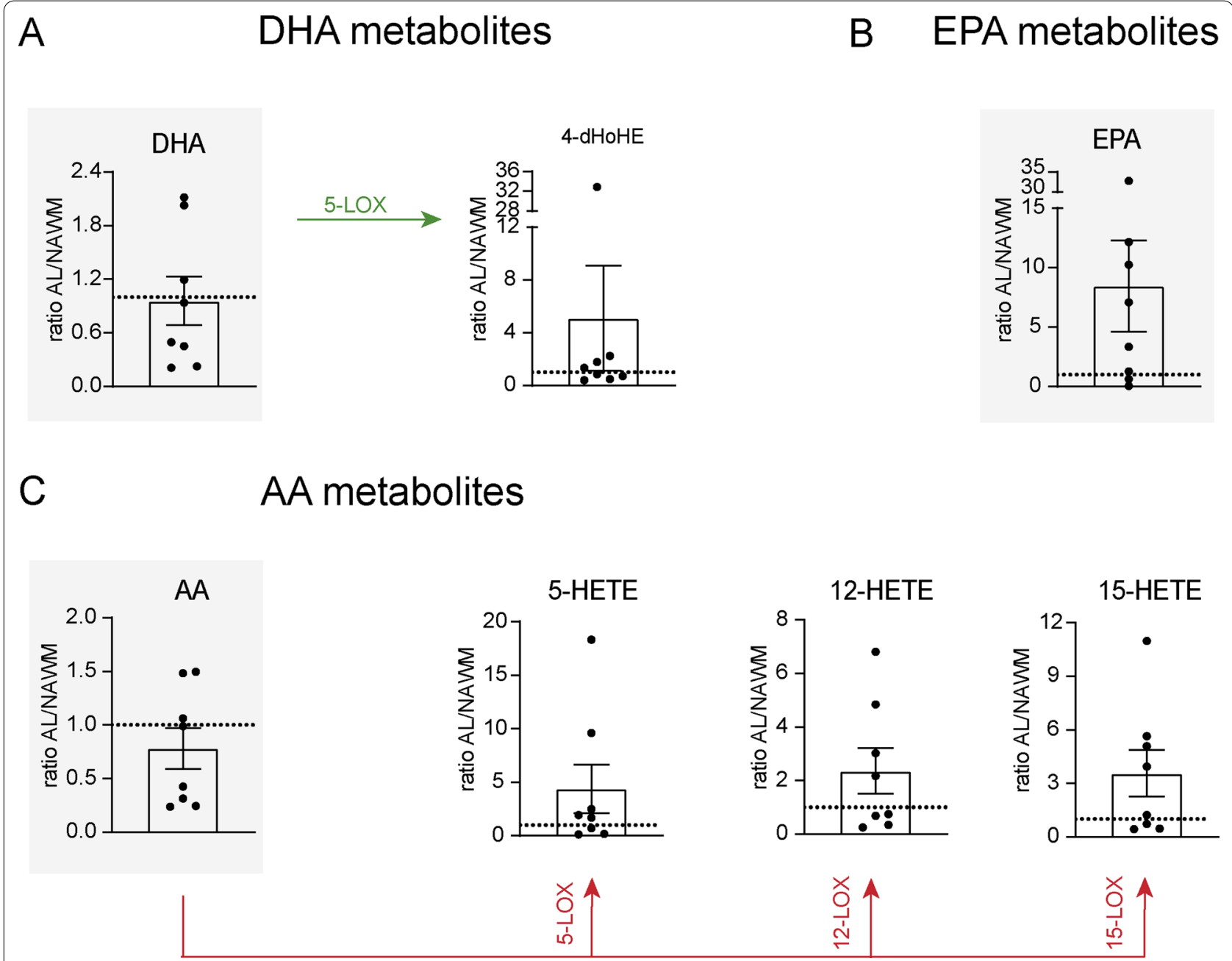

Fig. 4 Lipidomic analysis of SPM synthesis pathways markers in brain samples of MS patients. A-C Graphs showing the quantification of $\mathbf{A}$ DHA metabolome, B EPA metabolome and C AA metabolome in NAWM and AL $(n=8)$. Paired $t$-test. Data are shown as mean \pm SEM

These data suggest there is an imbalance between proinflammatory and pro-resolving lipid mediators in the spinal cord of EAE mice, in favour of the former,

\section{Synthesis of SPMs is impaired in MS patients}

We then performed metabolomic analysis on serum and brain samples of MS patients. These studies revealed that the serum levels of DHA, EPA and AA did not change between MS patients and healthy controls (Fig. 3A-C). Analysing the lipid mediators derived from the DHA metabolome in serum samples, we found that 14-HDoHE and 17-HDoHE, the maresin and resolvin D-series pathway markers, were increased in MS patients yet not significant (Fig. 3A). As for the EPA metabolome, the levels the RvE1 metabolic intermediate, 18-HEPE, as well as 12-HEPE and 15-HEPE were higher in MS patients, although only 15 -HEPE reached statistically significance (Fig. 3B). Furthermore, as for the AA metabolome, the levels of 11,12-EET, 5-HETE and 12-HETE were also increased in the serum of MS patients yet not significantly (Fig. 3C). Despite we found several SPM intermediate precursors derived from DHA, EPA and AA in the serum samples, SPMs were undetected in serum samples of MS patients and healthy donors.

We then performed metabolomic studies in active lesions (AL) and normal-appearing white matter (NAWM) of MS post-mortem brain tissue to investigate whether the synthesis of SPMs was defective at the specific regions of the CNS undergoing inflammation. We found that DHA, EPA and AA levels did not vary significantly in AL relative to NAWM, although half of MS individuals showed mark increase in EPA levels in AL (Fig. 4A-C). We also detected some intermediate precursors of SPM synthesis in brain MS samples, although no significant differences were found in AL as compared to NAWM (Fig. 4A-C). From the DHA metabolome, the 
only lipid mediator detected was the 4-HDoHE, a LOX-5 metabolite that in animal models has been shown to mediate the antiangiogenic effect of omega- 3 fatty acids [29]. However, the levels of 4-HDoHE in AL were higher in only 2 out $8 \mathrm{MS}$ patients, but no significant differences were found between AL and NAWM (Fig. 4A). Lipid mediators generated from the EPA metabolome were undetected in AL and NAWM. As for the AA metabolome, we found that 5-HETE, 12-HETE and 15-HETE levels in AL were higher in 4 out of 8 patients, but no significant changes were observed (Fig. 4C). Similar to serum samples, we did not detect any SPM in the MS brain AL and NAWM. Collectively, these data suggest that similar to EAE mice, the class switch from proinflammatory to pro-resolution lipid mediators does not occur properly in the periphery and in the CNS of MS patients.

\section{Feeble expression of ALOX12 and ALOX15 in MS patients and EAE mice}

Since our data suggest there is impaired activation of the resolution programmes of inflammation in MS patients
A

PBMC

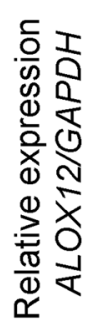

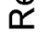

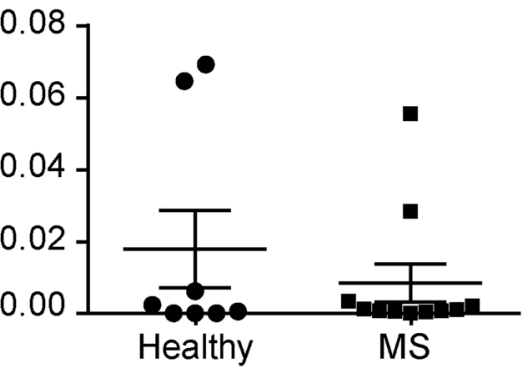

C

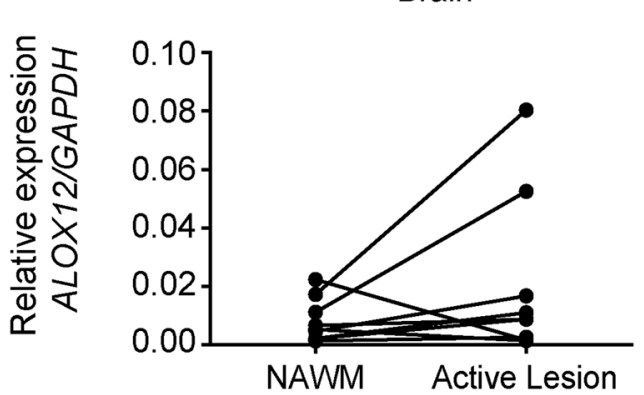

B

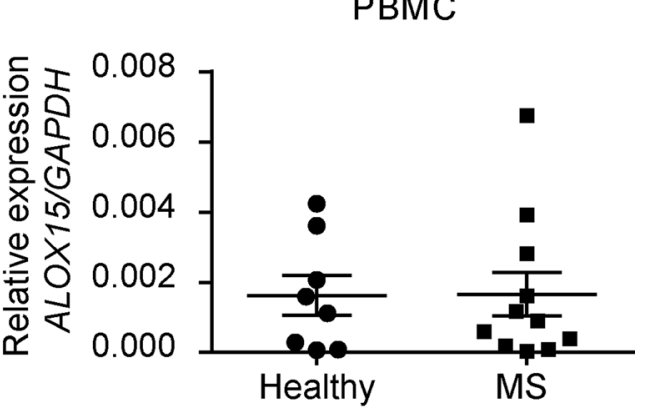

$\mathrm{D}$

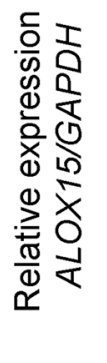

Brain

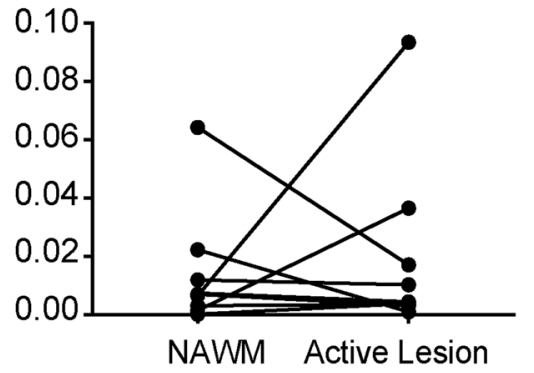

$E$

Spinal cord (mice)

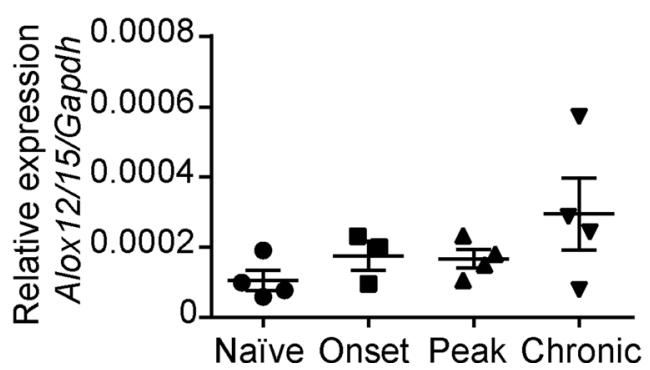

Fig. 5 Expression of lipoxygenase 12 and 15 in MS patients and lipoxygenase 12/15 in EAE mice. A-D Graphs showing the mRNA levels of ALOX12 $\mathbf{A}, \mathbf{C}$ and ALOX15 B, D in PBMCs of MS individuals $(n=11)$ and healthy donors $(n=8) \mathbf{B}, \mathbf{C}$ and in MS brain AL and NAWM C, D $(n=9)$. E Time course expression of Alox12/15 mRNA levels in the spinal cord of EAE mice over disease progression ( $n=3-4$ per time point). Unpaired $t$-test in $\mathbf{A}$-D and one-way ANOVA, Bonferroni's post hoc test in (E). Data are shown as mean \pm SEM 
and EAE mice, we then assessed whether this was associated with a failure in the expression of the key biosynthetic enzymes involved in SPM production. Thus, we measured the expression of lipoxygenase-12 (ALOX12) and lipoxygenase-15 (ALOX15) in PBMCs of active MS patients and healthy donors, as well as in active MS lesions in post-mortem brain tissue. qPCR analysis revealed that mRNA levels of ALOX12 and ALOX15 were undetected or found at very low levels PBMCs of either healthy donors or MS patients (Fig. 5A, B). Then, when assessing the expression of these enzymes in postmortem MS brain samples, we did not find significant changes in the expression within the lesioned areas compared to the NAWM, despite some variability across patients (Fig. 5C, D). Indeed, only 2 out of 9 patients showed increased expression of these enzymes in the AL compared to NAWM, while the rest did not show any change or a slight downregulation (1 patient in ALOX12 and 2 in ALOX15) (Fig. 5C, D).

We then studied whether this phenomenon also occurred in EAE, one of the most widely used animal models of MS. For this purpose, we analysed the expression of Alox 12/15, the murine orthologue of the previous mentioned enzymes in humans and the initiating enzyme in the SPM biosynthetic pathway. qPCR analysis revealed that, similar to MS patients, mRNA levels of Alox12/15 were barely found in the CNS of EAE mice, although the transcripts of this enzyme tended to be greater at the chronic stage of the disease (Fig. 5E).

Overall, these results suggest that the key enzymes involved in the production of SPMs are not induced in MS individuals nor EAE mice.

\section{Effects of MaR1 on inflammation in EAE}

Since we demonstrated that production of SPMs is not induced in MS patients and in EAE mice, we proceeded to investigate whether exogenous administration of SPMs attenuates inflammation in EAE. Among the different SPMs, we tested the effects of MaR1 since (i) this SPM was undetected in the serum and brain active lesions of MS patients; (ii) unlikely other SPMs, such as RvD1, RvD5 and NP1, MaR1 was undetected in the spinal cord of EAE mice; (iii) previous studies revealed that this SPM is also undetected in plasma [20] and CFS [6] samples of MS patients; (iv) MaR1, in contrast to other SPMs that were also undetected in MS and EAE patients, such as RvD2-RvD4, has already demonstrated efficacy in minimizing inflammation in the CNS [20]; and (v) the contribution of this SPM in demyelinating diseases is unknown. For this purpose, we administered $1 \mu \mathrm{g}$ to EAE mice, once a day from disease onset (0.5-1 EAE score) and we assessed the protein levels of 14 cytokines in the spinal cord at the disease peak (4-4.5 EAE score). Luminex

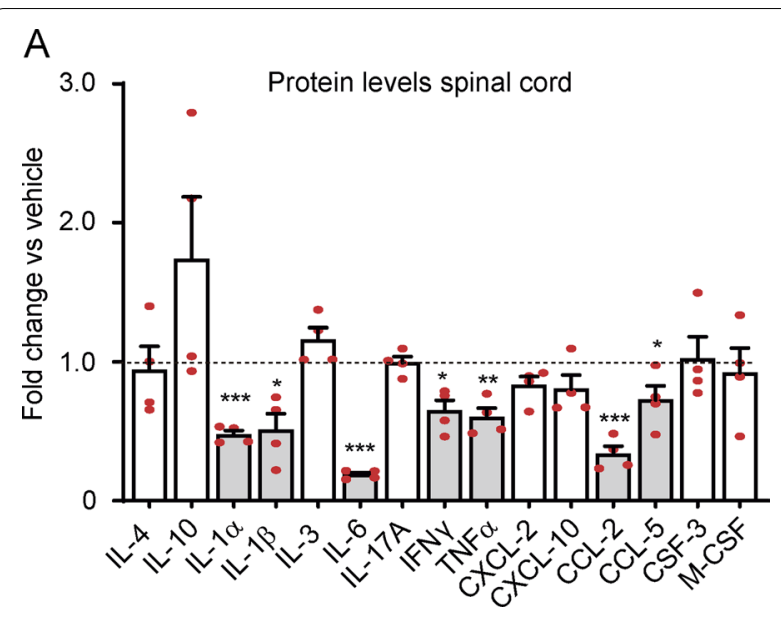

Fig. 6 MaR1 reduces pro-inflammatory cytokines in the spinal cord of EAE mice. Plot showing the fold change in cytokine protein levels of different cytokines in the spinal cord of EAE mice treated with MaR1 relative to those treated with vehicle (dotted line). Grey bars highlight those cytokines that are significantly regulated by MaR1. ${ }^{*} p<0.05 ;{ }^{* *} p<0.01 ;{ }^{* * *} p<0.001$ vs. vehicle. Unpaired $t$-test $(n=4$ per group). Data are shown as mean \pm SEM

assay revealed that MaR1 reduced significantly the levels of 5 out 9 pro-inflammatory cytokines (IL- $1 \alpha$, IL-1 $\beta$, IL-6, TNF $\alpha$ and INF $\gamma$ ) and 2 out the 4 chemokines measured (CCL-2, CCL-5) (Fig. 6; Additional file 1: Table S3).

Since cytokines and chemokines coordinate the migration of immune cells into tissues, we next studied whether MaR1 modulates the number of immune cells in the spinal cord of EAE mice at the peak of the disease. Flow cytometry experiments revealed that MaR1 strongly reduced the numbers of $\mathrm{T}$ cells, including both $\mathrm{CD} 4+\mathrm{T}$ cells $(\mathrm{CD} 3+, \mathrm{CD} 4+)$ and $\mathrm{CD} 8+$ cells $(\mathrm{CD} 3+$, CD8+), and B cells (CD45+, CD11b-, CD3-, CD24+). $\mathrm{MaR} 1$ also reduced the accumulation of macrophages (CD45high, CD11b,$+ \mathrm{F} 4 / 80+$ ) and activated microglial cells $(\mathrm{CD} 45 \mathrm{low}, \mathrm{CD} 11 \mathrm{~b}+)$ but not neutrophils (CD45high, CD11b + , F4/80-, Ly6G +) at this time point (Fig. 7A; Additional file 1: Fig. S1).

Due to the role of cytokines and SPMs in modulating $\mathrm{T}$ cell and macrophage phenotypes $[4,30]$, we investigated whether MaR1 affects these immune cells responses. We found that MaR1 reduced the number of CD4 T cells expressing IFN $\gamma$ in the spinal cord of EAE mice, suggesting that the number of pathogenic Th1 cells was reduced (Fig. 7B; Additional file 1: Fig. S2B). However, MaR1 did not alter the percentage of $\mathrm{T}$ cells expressing tBet, a transcription factor associated with Th1 responses (Fig. 7C). MaR1 did not attenuate the infiltration of Th2 nor Th17 cells since the expression signature cytokines and transcription factors, GATA3 and ROR $\gamma$, respectively, remained stable after treatment (Fig. 7B, C). However, 
MaR1 increased the proportion of Tr1 cells (CD3+, $\mathrm{CD} 4+, \mathrm{CD} 49 \mathrm{~b}+)$ (Fig. 7C; Additional file 1: Fig. S2B), which have a crucial role in maintaining tolerance to self-antigens, and increased in $\sim 50 \%$ the percentage of this lymphocyte subset producing IL-10, although not to the significant level (Fig. 7D; Additional file 1: Fig. S2C). MaR1 treatment, however, did not alter the percentage of Treg cells (CD3+, CD4+, FoxP3 +) (Fig. 7C; Additional file 1: Fig. S2C), another subset of regulatory lymphocytes, but it tended to increase their production of IL-10 (Fig. 6D; Additional file 1: Fig. S2C).

As for macrophages, we found that MaR1 reduced the accumulation of this myeloid cells in the spinal cord of EAE mice, but it increased the percentage of CD206 + macrophages (CD45high, CD11b +, F4/80+, CD206+) (Fig. 7A, E; Additional file 1: Fig. S3A), indicating that this SPM increased the proportion of antiinflammatory macrophages. In this line, we also found that MaR1 bolstered the ratio Ly6C $\mathrm{C}^{\text {low }} / \mathrm{Ly} 6 \mathrm{C}^{\text {high }}$ in macrophages by threefold, further supporting that this SPM favoured the anti-inflammatory responses of macrophages. (Fig. 5F; Additional file 1: Fig. S4B). Similar to macrophages, microglia counts were markedly reduced by MaR1 (Fig. 7A). However, this SPM led to increased proportion of anti-inflammatory CD206 + microglia (CD45low, CD11b+, CD206+) (Fig. 7G; Additional file 1: Fig. S3C).

We then assessed whether the reduction in leukocyte infiltration in the spinal cord by MaR1 was associated with a reduction in the expression of adhesion molecules in different population of immune cells. We found that MaR1 did not alter the expression of any of the studied adhesion molecules (CD11a, ICAM-1, VLA-4 and $\mathrm{CD} 62 \mathrm{~L}$ ) in macrophages, microglia, granulocytes or lymphocytes (Additional file 1: Fig. S4A-C), suggesting that the reduced infiltration of leukocytes into the CNS of EAE mice treated with MaR1 was likely related to the reduced expression of pro-inflammatory cytokines in the spinal cord rather than to an effect of MaR1 on leukocyte adhesion on blood vessels. Nonetheless, we did find an increase in the percentage of macrophages expressing VLA-4 (CD45high, CD11b +, F4/80+, VLA-4+) in MaR1-treated mice (Additional file 1: Fig. S4A), possibly associated with the enhanced infiltration of anti-inflammatory macrophages that we described before.
Next, we investigated whether the lessened leukocyte infiltration in the CNS after MaR1 treatment is linked with the attenuation of immune cells expansion in the lymph nodes or in the blood stream. We found that MaR1 slightly reduced the number of the different leukocyte populations in the lymph nodes, although the result was not statistically significant (Fig. 8A). However, MaR1 reduced by $\sim$ twofold the number of dendritic cells (CD45high, CD11b,$+ \mathrm{F} 4 / 80+, \mathrm{CD} 11 \mathrm{c}$ ), which have a decisive role in $\mathrm{T}$ cell priming. However, in contrast to that observed in the spinal cord, MaR1 did not modulate the phenotype of $\mathrm{CD} 4 \mathrm{~T}$ cells and monocytes. (Fig. 8C-D).

Despite the minimal actions of MaR1 on lymphocyte and monocyte in the lymph nodes, we observed fewer CD4 T cells, B cells and monocytes in the blood of EAE mice (Fig. 9A). MaR1 also led to changes in the phenotype of circulating CD4 $\mathrm{T}$ cells, increasing the percentage of $\operatorname{Tr} 1$ cells and also reducing that of pathogenic Th1 cells (Fig. 9B). Nonetheless, MaR1 did not modify the phenotype of circulating monocytes, which had a predominant pro-inflammatory state in both treated and no treated mice (CD45high, CD11b,$+ \mathrm{F} 4 / 80+$, Ly6C $^{\text {high }}$ ) (Fig. 9C). These results suggested that some immune cell responses observed in the spinal cord of MaR1-treated mice might be, in part, due to the actions of this SPM in the periphery.

\section{Systemic administration of MaR1 attenuates EAE}

To investigate whether the effects of MaR1 on inflammatory processes carry a clinical outcome, we assessed the effects of exogenous delivery of MaR1 on EAE symptoms. We found that mice treated with MaR1 were markedly protected against neurological impairments, despite the treatment being initiated at disease onset (data pooled from two independent experiments) (Fig. 10A, B). Indeed, MaR1-treated mice reached an average EAE score of $\sim 2.5$ by the end of the follow-up, while vehicletreated mice reached $\sim 4.2$ (Fig. 10A). Moreover, mice treated with MaR1 had significantly lower cumulative and maximum EAE scores than vehicle-treated mice (Fig. 10B).

We then evaluated whether the enhancement in neurological functions observed in EAE mice treated with MaR1 was related to preservation of myelin within the CNS. (Fig. 10C, D). However, histological sections

\footnotetext{
(See figure on next page.)

Fig. 7 MaR1 attenuates inflammation in the spinal cord of mice undergoing EAE. A-G Graph showing the quantification of different immune cell populations in the spinal cord $(\mathbf{A})$, the number of CD4 + T cells expressing IL-4, IL-10, IFNy or IL-17A (B), the percentage of CD4 + T cells expressing the CD49b, FoxP3, tBet, GATA3 or RORY (C), the percentage of regulatory lymphocytes expressing IL-10 (D), the percentage of macrophages expressing polarization markers $(\mathbf{E})$, the ratio of Ly $6 C^{\text {low }} /$ Ly $6 C^{\text {high }}$ macrophages $\mathbf{F}$ and the percentage of microglial cells expressing polarization markers in the spinal cord after MaR1 or vehicle treatment. ${ }^{*} p<0.05 ;{ }^{* *} p<0.01 ;{ }^{* * *} p<0.001$ vs. vehicle. Unpaired $t$-test ( $n=4$ per group). Data are shown as mean \pm SEM
} 


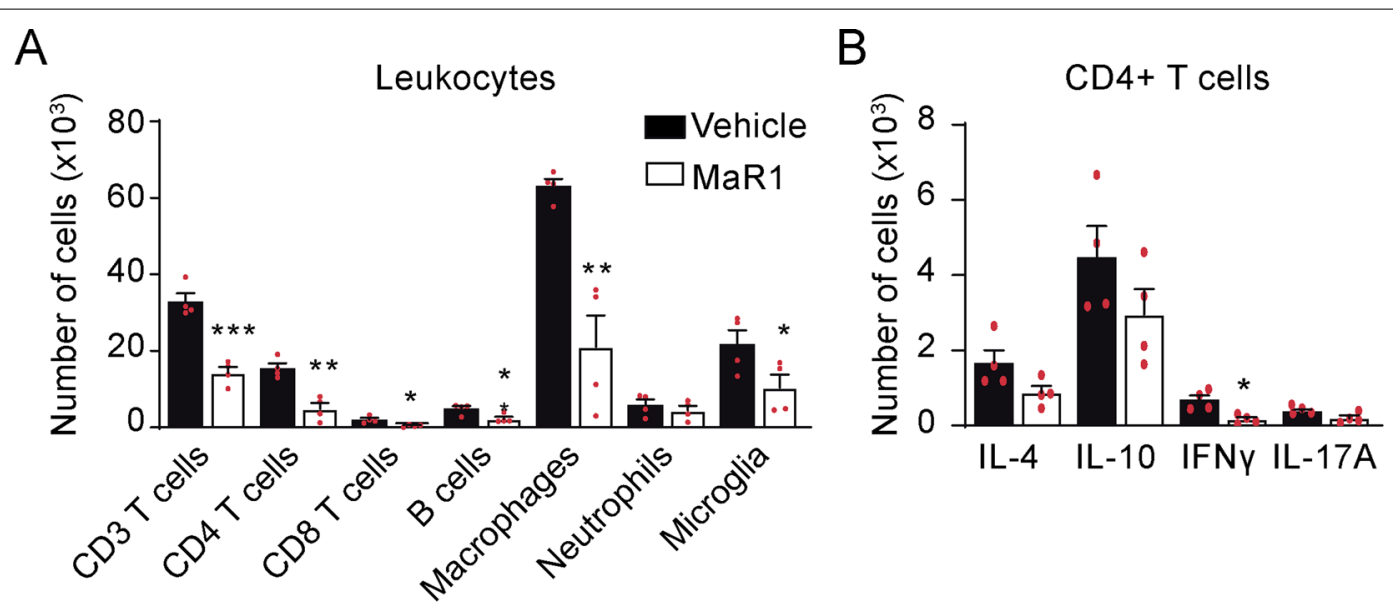

C

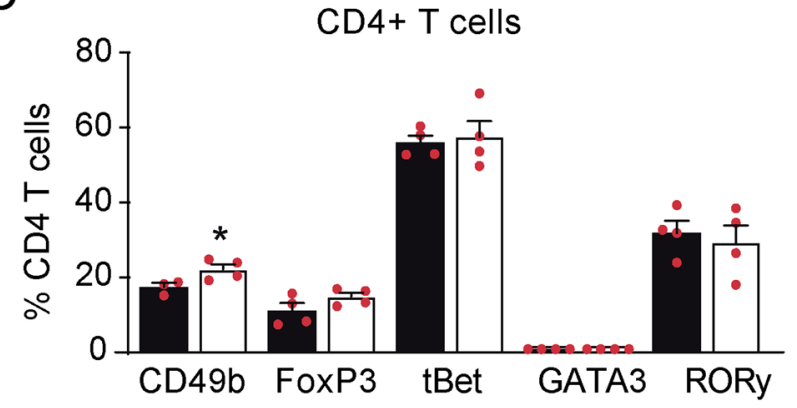

E

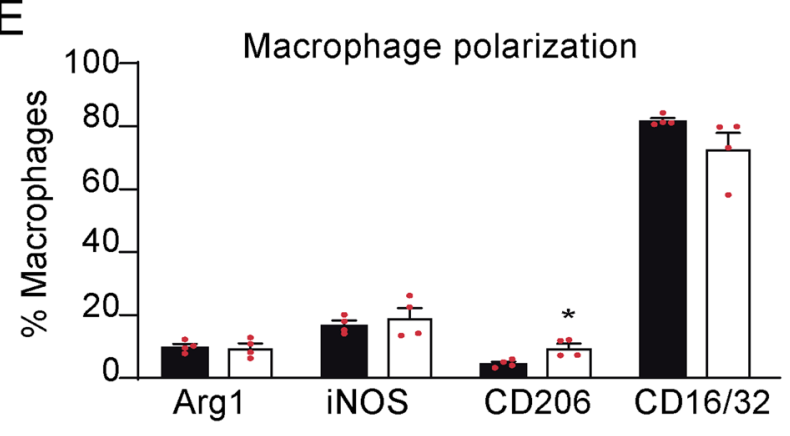

G

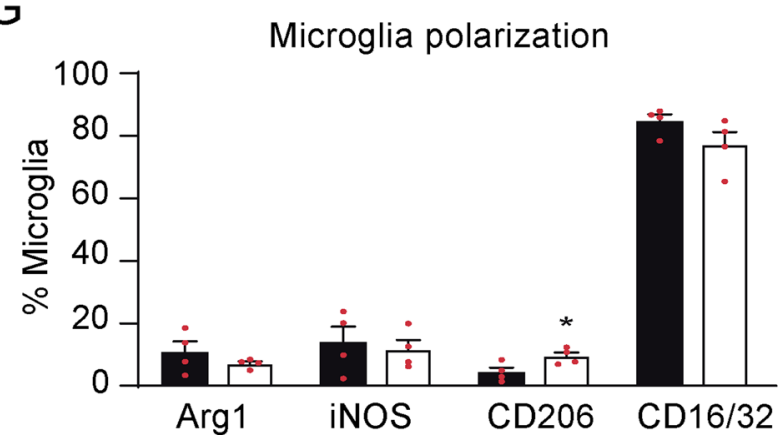

D

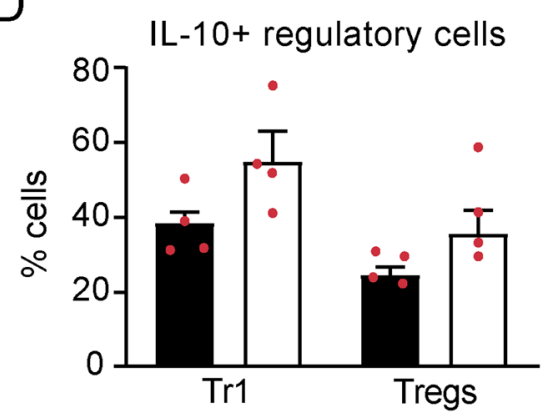

$\mathrm{F}$

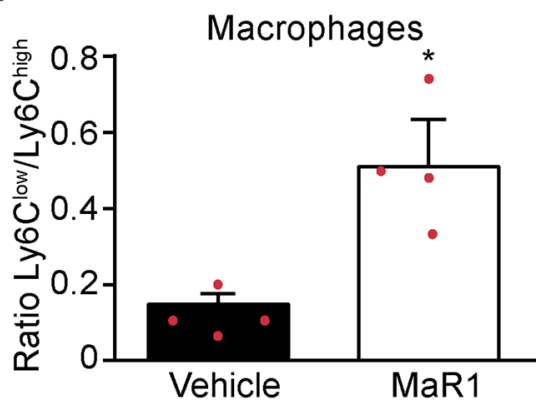

Fig. 7 (See legend on previous page.) 

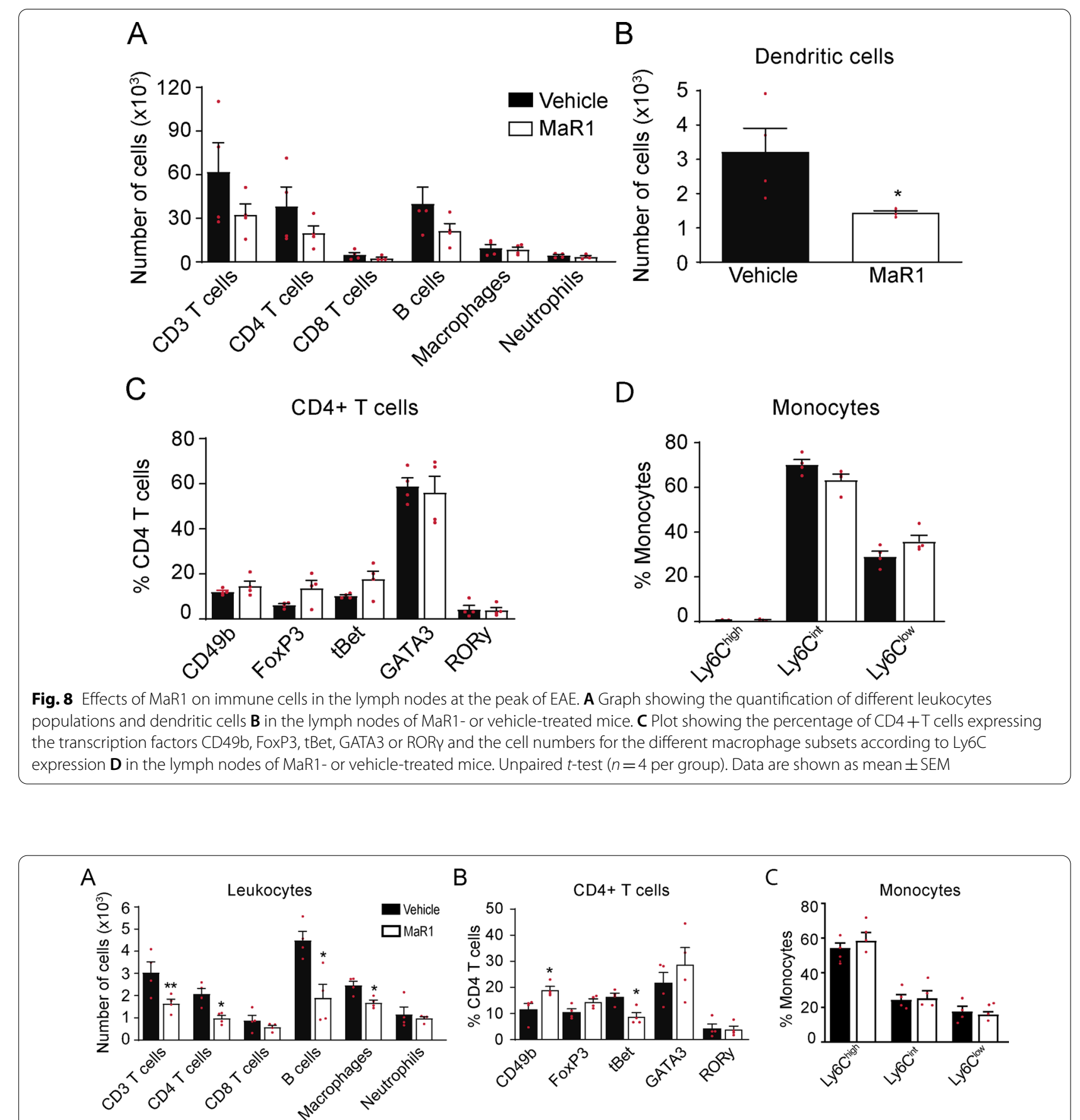

Fig. 9 Effects of MaR1 on immune cells in the blood of EAE mice at disease peak. A Graph showing the number of different leukocytes populations in the blood of MaR1- or vehicle-treated mice. B Plot showing the percentages of CD4 + T cells expressing the transcription factors CD49b, FoxP3, tBet, GATA3 or RORY and D different macrophage phenotypes according to Ly6C expression in blood of EAE mice treated with MaR1 or vehicle. Unpaired $t$-test $\left(n=4\right.$ per group). ${ }^{*} p<0.05 ;{ }^{* *} p<0.01$ vs. vehicle. Unpaired $t$-test ( $n=4$ per group). Data are shown as mean $\pm \operatorname{SEM}$

immunostained against NF revealed that there were very few axons within the demyelinating lesions of mice treated with MaR1 or vehicle (Additional file 1: Fig. S5). These data suggest that the reduced neurological deficits observed in EAE mice treated with MaR1 was due to the ability of this SPM to reduce the size of the CNS lesions rather than to remyelination. 

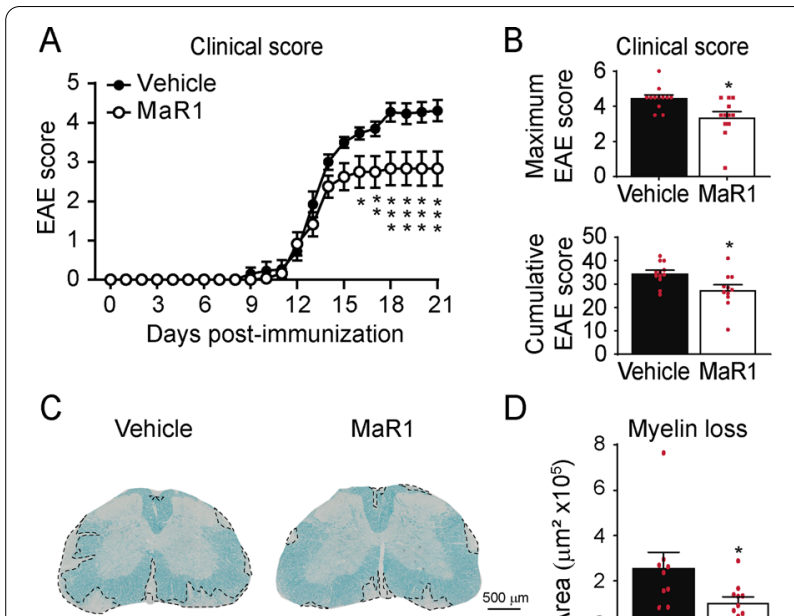

D

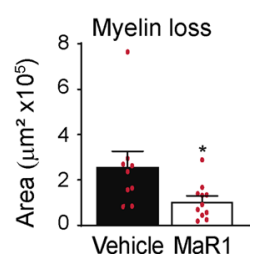

Fig. 10 Effects of MaR1 on neurological deficits and myelin loss in EAE mice. A, B Graphs showing the clinical score of EAE mice treated with MaR1 or vehicle over disease progression $(\mathbf{A})$, as well as the maximum and cumulative EAE score (B). C Graph showing the quantification of myelin loss in the lumbar spinal cord of MaR1- or vehicle-treated mice at 21 days post-induction. D Representative histological spinal cord tissue sections stained with LFB from EAE mice treated with vehicle and MaR1. ${ }^{*} p<0.05 ;{ }^{* *} p<0.01$; ${ }^{* * *} p<0.001$ vs. vehicle. Two-way ANOVA with repeated measures, Bonferroni's post hoc test in A ( $n=12$ per group). Unpaired t-test in B ( $n=12$ per group) and C ( $n=9$ in vehicle and $n=11$ in MaR1). Data were pooled from two different experiments. Data are shown as mean \pm SEM

Overall, these data reveal the therapeutic potential of MaR1 in attenuating neuroinflammation and protecting from tissue damage and clinical signs of EAE.

\section{Discussion}

In the present study, we report that the production of the main lipid mediators that activate the resolution programmes of inflammation is deficient in serum and active brain lesions of MS patients, as well as, in the CNS of EAE mice. These findings are associated with impaired induction of key enzymes involved in the biosynthetic pathways of SPMs. We also reveal that the administration of the resolution agonist, coined MaR1, in EAE mice attenuates the pro-inflammatory environment of the CNS, modulates $\mathrm{T}$ cell and macrophage responses towards a more regulatory or anti-inflammatory phenotype, and reduces the blood counts of different leukocyte populations. Importantly, we demonstrate that the effects of MaR1 on inflammation are associated with marked protection against neurological decline and myelin loss despite treatment initiation at disease onset.

PUFAs play a critical role in the regulation of inflammation by controlling several processes involved in its onset and termination $[2,31,32]$. In general, eicosanoids generated via the actions of cyclooxygenase 1 and
2 (COX-1 and 2) and 5-LOX from AA, such as prostaglandins, thromboxanes and leukotrienes, contribute to inflammation by promoting chemotaxis and activation of immune cells and increasing vascular permeability $[33,34]$. Nevertheless, not all the actions of eicosanoid are pro-inflammatory since a number of in vivo studies revealed that $15 \mathrm{~d}$-Prostaglandin $\mathrm{J} 2$, for instance, attenuates inflammation and neurological decline in EAE and spinal cord injury $[35,36]$.

In contrast, SPMs are key players in the resolution of inflammation. Except for lipoxins, SPMs are derived from omega-3 PUFA, in particular, from DHA and EPA. DHA can give rise to several resolution agonists, including those of the resolvin D series, protectin D and MaRs. EPA can also yield some SPMs, namely, resolvin E series $[2,7,9,11,37,38]$.

Omega-3 PUFAs are enriched in oils derived from fish and algae. Various epidemiological studies have indicated that omega-3 PUFA dietary supplementations are associated with improved clinical outcome of various pathologies, such as metabolic syndrome or cardiovascular diseases [39, 40]. In MS, however, the beneficial actions of omega-3 PUFA supplementation are inconsistent, as one trial failed to show clinical improvement despite the effectively increased omega-3 PUFA levels in patients as a result of the supplementation [41, 42]. Thus, it is very plausible that this lack of efficacy stems from the failure of MS patients to produce adequate amounts of SPMs, rather than from the bioavailability of omega-3 PUFA in their serum.

Previous studies have reported that lipid mediator pathways are dysregulated in various nervous systemrelated pathologies $[4,6,19,20,43]$. For instance, inappropriate SPM production has been observed in other CNS conditions, such as in Alzheimer's [43] and in mice after spinal cord contusion injury [4]. A recent report has uncovered that most SPMs are absent in plasma samples of MS patients at different stages of the disease, and only RvD1, RvD5 and NP1 could be detected [20]. These observations are in agreement with our findings shown here, although we were unable to detect any SPM in serum or inflammatory regions of the brain. These small differences between both studies could be due to type of sample analysed, and in case of the active brain lesions samples, to the limited amount of tissue available. However, RvD1, RvD5 and NPD1 were the three SPMs that we detected in the spinal cord of mice at physiological conditions, but their levels did not increase at different stages of EAE disease. Interestingly, we found that the impaired production of SPMs in serum plasma of MS patients was associated with deficient induction in the expression of the main SPM biosynthetic enzymes in PBMCs, which is in agreement with the work of Kooij and colleagues [20]. 
Besides, we also provided evidence indicating that this inefficient induction in 12- and 15-LOX expression also occurs in regions of the brain undergoing inflammation in MS patients, as well as in the spinal cord of EAE mice. Therefore, these results suggest that there is a failure in peripheral and central production of SPMs in MS, which may be responsible, in part, of the dysregulated deleterious inflammatory response characteristic of this disease.

Interestingly, Kooij and colleagues observed inverse correlation between the plasma levels of these RvD1, RvD5 and NP1 and clinical severity in MS patients [20], and that LXA4, LXB4, RvD1 and PD1 reduced MSderived monocyte activation and cytokine production, suggesting that administration of SPM could attenuate inflammation in MS patients. In this line, prophylactic administration of RvD1 in EAE alleviated the clinical signs of disease, suggesting that the exogenous administration of SPMs could revert the pathological signs of neuroinflammation [19].

MaR1 is an understudied member of the SPM superfamily $[9,44]$. There are just few studies demonstrating the beneficial actions of this SPM in the CNS. MaR1 not only enhanced inflammatory resolution after spinal cord injury and reduced myelin loss and neurological deficits [4], but also promoted neuroprotection in spinal muscular atrophy [45] and cerebral ischemia [46]. Thus, we complete this scenario since herein we provide data demonstrating that the administration of MaR1 in EAE mice also conferred protection against functional deficits and myelin loss, despite initiating the treatment after disease onset. We also found that MaR1 modulates different inflammatory mechanisms. First, MaR1 reduced the protein levels of different proinflammatory cytokines in the spinal cord of EAE mice, including IL- $1 \alpha$, IL-1 $\beta$, TNF $\alpha$, IFN $\gamma$ and IL-6, among others, but did not alter the amounts of anti-inflammatory/immunomodulatory cytokines, such as IL-4 or IL-10. These pro-inflammatory cytokines play a critical role in the development of EAE [47-49], and are associated with neurodegeneration and progression in MS $[50,51]$. Second, MaR1 reduced the accumulation of major leukocyte populations in the spinal cord of EAE mice, albeit not neutrophils. This may have occurred, at least partially, due to the decreased levels of cytokines in the spinal cord, and also because of MaR1's ability to hamper the accumulation of immune cells also into the blood. Third, MaR1 modulated the responses of macrophages and CD4 $\mathrm{T}$ cells towards a more anti-inflammatory profile. In particular, MaR1 reduced pathogenic Th1 cells in the spinal cord and increased the number of non-classical regulatory $\mathrm{T}$ cells. In agreement with our findings, a previous study revealed that resolution agonists, including MaR1, attenuate the activation of pathogenic Th1 and Th17 cells and their generation from naïve CD4 cells [30]. We did not observe any significant change in the cell number of different Th cell populations in the lymph nodes, suggesting that MaR1 does not regulate the peripheral activation of Th cells in EAE, at least when its administration occurs after disease onset. MaR1, however, reduced Th1 cell number in the blood and increased that of non-classical Tregs. In this study, we also observed that macrophages present in the spinal cord of EAE adopted a more anti-inflammatory phenotype, similar to our previous findings in spinal cord injury [4]. Because this was only observed in the CNS and not also in the lymph nodes or blood, it suggests that the modulation of the inflammatory environment of the CNS by MaR1 is most likely to be the responsible for the effects on macrophages. However, the impact of MaR1 on immune cell numbers is unlikely dependent on the modulation of adhesion molecules in peripheral leukocytes or microglial cells since this SPM fail to reduce their expression. Indeed, MaR1 increased the expression of VLA-4 in macrophages. This cell adhesion has been recently shown to be important for the infiltration of Ly6 $\mathrm{C}^{\text {low }}$ macrophages [52] and may account for the higher infiltration of anti-inflammatory macrophages observed in the CNS of EAE mice treated with MaR1.

\section{Conclusions}

Overall, we provide clear evidence that there is inefficient production of SPMs in the spinal cord of EAE mice as well as in the CNS and periphery of MS patients. Our data indicate that exogenous administration of MaR1 is effective at enhancing multiple mechanisms needed for preventing inflammation in EAE mice and confers protection against neurological decline and myelin loss. These data therefore indicate that SPMs in general, and MaR1 in particular, represent a novel therapeutic avenue for the treatment of MS with promising potential.

\footnotetext{
Abbreviations

AA: Arachidonic acid; CFA: Complete Freund's adjuvant; CNS: Central nervous system; DHA: Docosahexaenoic acid; EAE: Experimental autoimmune encephalomyelitis; EPA: Eicosapentaenoic acid; LOX: Lipoxygenase; LX: Lipoxins; MaR1: Maresin-1; MS: Multiple sclerosis; NAWM: Normal-appearing white matter; PBMCs: Peripheral blood mononuclear cells; PBS: Phosphate-buffered saline; PD/NPD: Protectins/Neuroprotectins; PPMS: Primary-progressive multiple sclerosis; PUFAs: Polyunsaturated fatty acids; qPCR: Real-time Quantitative PCR Assay; RRMS: Relapsing-remitting multiple sclerosis; RvD: Resolvin D; RvE: Resolvin E series; sMRM: Scheduled multiple reaction monitoring; SPMS: Secondary-progressive multiple sclerosis; SPMs: Specialized pro-resolving lipid mediators.
} 


\section{Supplementary Information}

The online version contains supplementary material available at https://doi. org/10.1186/s12974-022-02386-1.

Additional file 1. Fig. S1. MaR1 reduces the accumulation of immune cells in the spinal cord of mice at the peak of EAE. Fig. S2. MaR1 modulates $T$ cells responses in the spinal cord of EAE mice. Fig. S3. MaR1 modulates the macrophages and microglia phenotypes towards a more anti-inflammatory phenotype. Fig. S4. Effects of the treatment with MaR1 in the expression of adhesion molecules on immune cells in the spinal cord at the peak of EAE. Fig. S5. Effects of MaR1 in axonal damage in the lesioned areas of the spinal cord at the peak of EAE. Table S1. Descriptive information about human samples of MS patients and healthy controls used for QPCR analysis. Table S2. Descriptive information about human samples of MS patients and healthy controls used for lipidomic analysis. Table S3. Cytokine expression in the spinal cord of naïve, vehicle- and MaR1-treated mice at the peak of EAE.

\section{Acknowledgements}

The authors thank Mónica Espejo, Jessica Jaramillo, Neus Hernández and Manuela Costa for their technical assistance.

\section{Authors' contribution}

RL-V designed the study, analysed and interpreted the data, and wrote the manuscript. AS-F collected murine samples, performed the experiments, analysed and interpreted the data, and wrote the manuscript. SZ collected human samples, interpreted data and reviewed the manuscript. MC assisted in the collection of human samples and reviewed the manuscript. MM and HR performed the metabololipidomic analyses in human and murine samples and reviewed the manuscript. AP provided human samples. All authors read and approved the final manuscript.

\section{Funding}

This work was supported by grants from Spanish Ministry of Economy and Competitiveness (SAF2016-79774-R), Spanish Ministry of Science and Innovation (PID2020-120267RB-100/AEI/1013039/501100011033), Wings for Life International Foundation, Red de Terapia Celular (TERCEL), funds from "la Caixa" Foundation under agreement LCF/TR/CI17/10020018 and LCF/ PR/HA17/52170001 to R.L-V, and by funds from CSIC Grupos Universidad de la República, Uruguay (Grant 536) to HR. A.P. holds the T1 (senior) Canada Research Chair in MS.

\section{Availability of data and materials}

All the data generated or analysed during this study are included in this published article ant its supplementary information files.

\section{Declarations}

\section{Ethics approval and consent to participate}

Tissue samples were collected from MS patients with full ethical approval (BH07.001) and informed consent was approved by the local ethics committee in Montreal (Canada). All experimental procedures were approved by the Universitat Autònoma de Barcelona Animal Experimentation Ethical Committee (CEEAH 2878) and followed the European Communities Council Directive 2010/63/EU, and the methods were carried out in accordance with the approved guidelines.

\section{Consent for publication}

Not applicable.

\section{Competing interests}

A.S.-F and R.L.-V. are inventors on a patent application filled by Universitat Autònoma de Barcelona "Specialized pro-resolving lipid mediators for use in the treatment of neurodegenerative diseases and/or autoimmune diseases" (PCT EP2018/051076). The remaining authors declare no competing financial interests.

\section{Author details}

${ }^{1}$ Institut de Neurociencies and Departament de Biologia Cel lular, Fisiologia i Immunologia, Facultat de Medicina, Universitat Autonoma de Barcelona, 08193 Bellaterra, Catalonia, Spain. ${ }^{2}$ Centro de Investigación Biomédica en Red de Enfermedades Neurodegenerativas (CIBERNED), Barcelona, Spain. ${ }^{3}$ Department of Neuroscience, Faculty of Medicine, Université de Montréal and Neuroimmunology Unit, Centre de Recherche du CHUM (CRCHUM), Montréal, Québec, Canada. ${ }^{4}$ Departamento de Bioquímica, Facultad de Medicina and Centro de Investigaciones Biomédicas (CEINBIO), Universidad de La República, Montevideo, Uruguay.

Received: 17 September 2021 Accepted: 13 January 2022

Published online: 02 February 2022

\section{References}

1. Russell P. Pathologic basis of disease. Pathology. 2007;7:260.

2. Serhan CN. Pro-resolving lipid mediators are leads for resolution physiology. Nature. 2014;510:92-101.

3. Serhan CN, Chiang N, Dalli J, Levy BD. Lipid mediators in the resolution of inflammation. Cold Spring Harb Perspect Biol. 2015;7(2):a016311.

4. Francos-Quijorna I, Santos-Nogueira E, Gronert K, Sullivan AB, Kopp MA, Brommer $\mathrm{B}$, et al. Maresin 1 promotes inflammatory resolution, neuroprotection, and functional neurological recovery after spinal cord injury. J Neurosci. 2017;37:11731-43.

5. López-Vales R, David S. Bioactive lipids in inflammation after central nervous system injury. In: Trostchansky A, Rubbo H, editors. Bioactive lipids in health and disease advances in experimental medicine and biology, vol. 1127. Springer: Cham; 2019. p. 181-94.

6. Prüss H, Rosche B, Sullivan AB, Brommer B, Wengert O, Gronert K, et al. Proresolution lipid mediators in multiple sclerosis_differential, disease severity-dependent synthesis - a clinical pilot trial, Bozza PT. PLoS ONE. 2013;8:e55859.

7. Schwab JM, Chiang N, Arita M, Serhan CN. Resolvin E1 and protectin D1 activate inflammation-resolution programmes. Nature. 2007;447:869-74.

8. Ruiz F, Vigne S, Pot C. Resolution of inflammation during multiple sclerosis. Semin Immunopathol. 2019;41:711-26.

9. Serhan CN, Yang R, Martinod K, Kasuga K, Pillai PS, Porter TF, et al. Maresins: novel macrophage mediators with potent antiinflammatory and proresolving actions. J Exp Med. 2009;206:15-23.

10 Chiang N, Serhan CN. Structural elucidation and physiologic functions of specialized pro-resolving mediators and their receptors. Mol Aspects Med. 2017;58:114-29.

11. Serhan CN. Treating inflammation and infection in the 21st century: new hints from decoding resolution mediators and mechanisms. FASEB J. 2017;31:1273-88

12. Wang L, Wang F-S, Gershwin ME. Human autoimmune diseases: a comprehensive update. J Intern Med. 2015;278:369-95.

13. Bhise V, Dhib-Jalbut S. Further understanding of the immunopathology of multiple sclerosis: impact on future treatments. Expert Rev Clin Immunol. 2016;12:1069-89.

14. Dendrou CA, Fugger L, Friese MA. Immunopathology of multiple sclerosis. Nat Rev Immunol. 2015;15:545-58.

15 Popescu BFG, Lucchinetti CF. Immunopathology. Handb Clin Neurol. 2016. https://doi.org/10.1016/B978-0-444-63432-0.00006-2.

16. Prat $A$, Antel J. Pathogenesis of multiple sclerosis. Curr Opin Neurol. 2005;18:225-30.

17. Lassmann H, Brück W, Lucchinetti CF. The immunopathology of multiple sclerosis: An overview. Brain Pathol. 2007;17:210-8.

18. Prineas JW, Kwon EE, Cho ES, Sharer LR, Barnett MH, Oleszak EL, et al. Immunopathology of secondary-progressive multiple sclerosis. Ann Neurol. 2001;50:646-57.

19. Poisson LM, Suhail H, Singh J, Datta I, Denic A, Labuzek K, et al. Untargeted plasma metabolomics identifies endogenous metabolite with drug-like properties in chronic animal model of multiple sclerosis. J Biol Chem. 2015;290:30697-712.

20. Kooij G, DeradaTroletti C, Leuti A, Norris PC, Riley I, Albanese M, et al. Specialized pro-resolving lipid mediators are differentially altered in peripheral blood of patients with multiple sclerosis and attenuate 
monocyte and blood-brain barrier dysfunction. Haematologica. 2019;105(8):2056-70.

21. DeradaTroletti C, Enzmann G, Chiurchiù V, Kamermans A, Tietz SM, Norris PC, et al. Pro-resolving lipid mediator lipoxin A4 attenuates neuro-inflammation by modulating $T$ cell responses and modifies the spinal cord lipidome. Cell Rep. 2021;35:109201.

22. Polman $\mathrm{CH}$, Reingold SC, Banwell B, Clanet M, Cohen JA, Filippi M, et al. Diagnostic criteria for multiple sclerosis: 2010 Revisions to the McDonald criteria. Ann Neurol. 2011:69:292-302.

23. Dhaeze T, Tremblay L, Lachance C, Peelen E, Zandee S, Grasmuck C, et al. CD70 defines a subset of proinflammatory and CNS-pathogenic $\mathrm{TH} 1$ / $\mathrm{TH} 17$ lymphocytes and is overexpressed in multiple sclerosis. Cell Mol Immunol. 2019;16:652-65.

24. Kuhlmann T, Ludwin S, Prat A, Antel J, Brück W, Lassmann H. An updated histological classification system for multiple sclerosis lesions. Acta Neuropathol. 2017:133:13-24.

25. Arbour N, Holz A, Sipe JC, Naniche D, Romine JS, Zyroff J, et al. A new approach for evaluating antigen-specific $T$ cell responses to myelin antigens during the course of multiple sclerosis. J Neuroimmunol. 2003:137:197-209.

26. Livak KJ, Schmittgen TD. Analysis of relative gene expression data using real-time quantitative $P C R$ and the $2-\triangle \triangle C T$ method. Methods. 2001;25:402-8.

27. Mastrogiovanni M, Ifrán E, Trostchansky A, Rubbo H. Lipidomic analysis of oxygenated polyunsaturated fatty acid-derived inflammatory mediators in neurodegenerative diseases. Biochem Soc Trans. 2011;39(5):1240-6.

28 Amo-Aparicio J, Martínez-Muriana A, Sánchez-Fernández A, López-Vales R. Neuroinflammation Quantification for Spinal Cord Injury. Curr Protoc Immunol. 2018;123:1-15.

29. Sapieha P, Stahl A, Chen J, Seaward MR, Willett KL, Krah NM, et al. 5-Lipoxygenase metabolite 4-HDHA is a mediator of the antiangiogenic effect of $\omega$-3 polyunsaturated fatty acids. Sci Transl Med. 2011. https://doi. org/10.1126/scitransImed.3001571.

30. Chiurchiù V, Leuti A, Dalli J, Jacobsson A, Battistini L, Maccarrone M, et al. Proresolving lipid mediators resolvin D1, resolvin D2, and maresin 1 are critical in modulating T cell responses. Sci Transl Med. 2016. https://doi. org/10.1126/scitransImed.aaf7483.

31. David S, Greenhalgh AD, López-Vales R. Role of phospholipase A 2s and lipid mediators in secondary damage after spinal cord injury. Cell Tissue Res. 2012;349:249-67.

32. Robinson DR. Lipid mediators of inflammation. Rheum Dis Clin North Am. 1987:13:385-405

33. Belury MA, Harris WS. Omega-6 fatty acids, inflammation and cardiometabolic health: Overview of supplementary issue. Prostaglandins, Leukot Essent Fat Acids. 2018;139:1-2.

34. Dennis EA, Norris PC. Eicosanoid storm in infection and inflammation. Nat Rev Immunol. 2015;15:511-23.

35. Diab A, Deng C, Smith JD, Hussain RZ, Phanavanh B, Lovett-Racke AE, et al. Peroxisome proliferator-activated receptor-agonist 15-Deoxy12,1412,14-prostaglandin J2 ameliorates experimental autoimmune encephalomyelitis. J Immunol. 2002;168:2508-15.

36. Kerr BJ, Girolami El, Ghasemlou N, Jeong SY, David S. The protective effects of 15-deoxy- $\triangle$-12,14-prostaglandin J2 in spinal cord injury. Glia. 2008:56:436-48.

37 Buckley CD, Gilroy DW, Serhan CN. Proresolving lipid mediators and mechanisms in the resolution of acute inflammation. Immunity. 2014:40:315-27.

38. Serhan CN, Chiang N. Endogenous pro-resolving and anti-inflammatory lipid mediators: a new pharmacologic genus. Br J Pharmacol. 2009:153:S200-15.

39. de Camargo TL, de Oliveira EP, Moreto F, Portero-McLellan KC, Burini RC. Omega-3 fatty acids supplementation decreases metabolic syndrome prevalence after lifestyle modification program. J Funct Foods Elsevier. 2015:19:922-8

40 Marik PE, Varon J. Omega-3 dietary supplements and the risk of cardiovascular events: a systematic review. Clin Cardiol. 2009:32:365-72.

41. Riccio P, Rossano R, Larocca M, Trotta V, Mennella I, Vitaglione P, et al. Antiinflammatory nutritional intervention in patients with relapsing-remitting and primary-progressive multiple sclerosis: a pilot study. Exp Biol Med. 2016:241:620-35.
42. Torkildsen O, Wergeland S, Bakke S, Beiske AG, Bjerve KS, Hovdal H, et al. $\omega-3$ fatty acid treatment in multiple sclerosis (OFAMS Study). Arch Neurol. 2012:69:1044-51.

43. Wang X, Zhu M, Hjorth E, Cortés-Toro V, Eyjolfsdottir H, Graff C, et al. Resolution of inflammation is altered in Alzheimer's disease. Alzheimer's Dement. 2015;11(40-50):e1-2.

44. Serhan CN, Dalli J, Colas RA, Winkler JW, Chiang N. Protectins and maresins: New pro-resolving families of mediators in acute inflammation and resolution bioactive metabolome. Biochim Biophys Acta. 2015;1851:397-413.

45. Ohuchi K, Ono Y, Joho M, Tsuruma K, Ogami S, Yamane S, et al. A Docosahexaenoic acid-derived pro-resolving agent, maresin 1, protects motor neuron cells death. Neurochem Res. 2018:43:1413-23.

46. Xian W, Li T, Li L, Hu L, Cao J. Maresin 1 attenuates the inflammatory response and mitochondrial damage in mice with cerebral ischemia/ reperfusion in a SIRT1-dependent manner. Brain Res. 2019;1711:83-90.

47. Lévesque SA, Paré A, Mailhot B, Bellver-Landete V, Kébir H, Lécuyer M-A, et al. Myeloid cell transmigration across the CNS vasculature triggers IL-1 $\beta$-driven neuroinflammation during autoimmune encephalomyelitis in mice. J Exp Med. 2016;213:929-49.

48. Schrijver HM, Van As J, Crusius JBA, Dijkstra CD, Uitdehaag BMJ. Interleukin (IL)-1 gene polymorphisms: Relevance of disease severity associated alleles with IL-1 $\beta$ and IL-1 ra production in multiple sclerosis. Mediators Inflamm. 2003;12:89-94.

49. Serada S, Fujimoto M, Mihara M, Koike N, Ohsugi Y, Nomura S, et al. IL-6 blockade inhibits the induction of myelin antigen-specific Th17 cells and Th1 cells in experimental autoimmune encephalomyelitis. Proc Natl Acad Sci. 2008;105:9041-6.

50. Kallaur AP, Oliveira SR, Simao ANC, De Almeida ERD, Morimoto HK, Lopes $J$, et al. Cytokine profile in relapsing-remitting Multiple sclerosis patients and the association between progression and activity of the disease. Mol Med Rep. 2013;7:1010-20

51. Rossi S, Motta C, Studer V, Macchiarulo G, Volpe E, Barbieri F, et al. Interleukin-1 $\beta$ causes excitotoxic neurodegeneration and multiple sclerosis disease progression by activating the apoptotic protein $\mathrm{p} 53$. Mol Neurodegener. 2014;9:56

52. Sun D, Zhang M, Sun P, Liu G, Strickland AB, Chen Y, et al. VCAM1/NLA4 interaction mediates Ly6Clow monocyte recruitment to the brain in a TNFR signaling dependent manner during fungal infection. PLOS Pathog. 2020;16:e1008361.

\section{Publisher's Note}

Springer Nature remains neutral with regard to jurisdictional claims in published maps and institutional affiliations.

Ready to submit your research? Choose BMC and benefit from:

- fast, convenient online submission

- thorough peer review by experienced researchers in your field

- rapid publication on acceptance

- support for research data, including large and complex data types

- gold Open Access which fosters wider collaboration and increased citations

- maximum visibility for your research: over 100M website views per year

At BMC, research is always in progress.

Learn more biomedcentral.com/submissions 